\title{
A New Class of Generalized Weibull-G Family of Distributions: Theory, Properties and Applications
}

\author{
Baitshephi Mashabe ${ }^{1}$, Broderick O. Oluyede ${ }^{2}$, Adeniyi F. Fagbamigbe ${ }^{1,3}$, Boikanyo Makubate ${ }^{1} \&$ Syamala \\ Krishnannair $^{4}$ \\ ${ }^{1}$ Department of Mathematics and Statistical Sciences, Botswana International University of Science and Technology, \\ Palapye, BW \\ ${ }^{2}$ Department of Mathematical Sciences, Georgia Southern University, Statesboro, GA, 30460, USA \\ ${ }^{3}$ Department of Epidemiology and Medical Statistics, College of Medicine, University of Ibadan, Nigeria \\ ${ }^{4}$ Department of Mathematical Sciences, University of Zululand, KwaDlangezwa 3886, South Africa \\ Correspondence: B. O. Oluyede, Department of Mathematical Sciences, Georgia Southern University, Statesboro, GA, \\ 30460, USA. E-mail: boluyede@georgiasouthern.edu
}

\author{
Received: August 23, 2018 Accepted: December 3, 2018 Online Published: December 9, 2018 \\ doi:10.5539/ijsp.v8n1p73 URL: https://doi.org/10.5539/ijsp.v8n1p73
}

\begin{abstract}
We propose a generalized class of distributions called the Webull-G Power Series (WGPS) family of distributions and its sub-model Weibull-G logarithmic (WGL) distributions. Structural properties of the WGPS family of distributions and its sub-model WGL distribution including hazard function, moments, conditional moments, order statistics, Rényi entropy and maximum likelihood estimates are derived. A simulation study to examine the bias, mean square error of the maximum likelihood estimators for each parameter is presented. Finally, real data examples are presented to illustrate the applicability and usefulness of the proposed model.
\end{abstract}

Keywords: Weibull distribution, power series, logarithmic distribution, maximum likelihood estimation

\section{Introduction}

There has been tremendous interest in the generalization or modification of the well-known classical distributions in order to provide better and flexible models for different real life applications. In several areas such as reliability, finance and insurance, there is need for generalized form of these distributions. This has led to several new methods for generating generalized new families of distributions.

Weibull distribution is not ideal for modeling non-monotone hazard rates, since in real life applications, empirical hazard rate curves often exhibit non-monotonic shapes such as a bathtub, upside-down bathtub (uni-modal) and others, thus the need for generating new families of distributions that can provide more flexibility in general situations including lifetime modeling.

There are several relatively new generalized distributions in the literature including the exponential-power series (EPS) distribution (Chahkandi \& Ganjali (2009)), Weibull-power series (WPS) distribution (Morais \& Barreto-Souza (2011)), generalized exponential-power series (GEPS) distribution (Mahmoudi \& Jafari (2012)), complementary exponential power series (Flores et al. (2013)), extended Weibull-power series (EWPS) distribution (Silva et al. (2013)), double bounded Kumaraswamy power series (Bidram \& Nekoukhou (2013)), Burr XII power series (Silva and Cordeiro (2015)), generalized linear failure rate-power series (GLFRPS) distribution (Alamatsaz and Shams (2016)), Birnbaum-Saunders power series distribution (Bourguignon, Silva \& Cordeiro (2014)), linear failure rate-power series (LFRPS) distribution (Mahmoudi and Jafari (2017)), and complementary extended Weibull-power series (CEWPS) distribution (Cordeiro \& Silva (2014)). Also, similar techniques were used by Oluyede et al. (2016), Roman et al. (2012), Lu \& Shi (2011), and Louzada, Marchi \& Roman (2014). Oluyede et al. (2018) developed a new Burr XII-Weibull-Logarithmic distribution for survival and lifetime data analysis. For compounding continuous distributions with discrete distributions, Nadarajah, Popović \& Ristić (2013) introduced the package Compounding in R software (R Development Core Team, (2014)).

We propose a new class of generalized distributions called the Weibull-G power series (WGPS) family of distributions and its sub-model Weibull-G logarithmic family of distributions. We compound the Weibull-G family and power series distributions, and introduce a new class of distributions and its sub-model called the Weibull-G logarithmic (WGL) family of distributions.

This content of this paper is organized as follows. In section 2, the generalized distribution, it's corresponding probability 
density function (pdf) and sub-models are given. Some structural properties including the hazard function, quantile function, and various sub-models, moments, moment generating function, conditional moments, the distribution of the order statistics, L-moments, Rényi entropy and estimates of model parameters are developed. In section 3, the special case of the Weibull-G Logarithmic distribution is presented. Monte Carlo simulation study is conducted to examine the bias and mean square error of the maximum likelihood estimators for each parameter in section 4. Applications of the proposed model to real data are given in section 5 , followed by summary remarks.

\section{The Model, Sub-models and Properties}

The generalized family of distributions called the Weibull-G power series (WGPS) family of distributions and some of its properties including expansion of the density, hazard function, quantile function and sub-models, moments, conditional moments and maximum likelihood estimation of model parameters are derived.

Bourguignon, Silva \& Cordeiro (2014) proposed the Weibull-G (WG) family of distributions with the cumulative distribution function (cdf) and probability density function (pdf) given by

$$
F(x ; \alpha, \beta, \xi)=1-e^{-\alpha\left[\frac{G(x ; \xi)}{G(x, \xi)}\right]^{\beta}},
$$

and

$$
f(x ; \alpha, \beta, \xi)=\alpha \beta g(x ; \xi)\left[\frac{G(x ; \xi)^{\beta-1}}{\bar{G}(x ; \xi)^{\beta+1}}\right] e^{-\alpha\left[\frac{G(x ; \xi)}{G(x ; \xi)}\right]^{\beta}},
$$

respectively, for $\alpha>0, \beta>0$, and $x \in \mathbb{R}$, where $G(x ; \xi)$ and $g(x ; \xi)$ are the cdf and pdf of any baseline distribution that depend on a parameter vector $\xi$ and $\bar{G}(x ; \xi)=1-G(x ; \xi)$.

We develop a generalization or an extension of the WG family of distributions by compounding it with the power series class of distributions. Let $\mathrm{N}$ be a zero truncated discrete random variable having a power series distribution, whose probability mass function (pmf) is given by

$$
P(N=n)=\frac{a_{n} \theta^{n}}{C(\theta)}, n=1,2,3, \ldots,
$$

where $C(\theta)=\sum_{n=1}^{\infty} a_{n} \theta^{n}$ is finite, $\theta>0$ and $\left\{a_{n}\right\}_{n \geq 1}$ a sequence of positive real numbers. The power series family of distributions includes binomial, Poisson, geometric and logarithmic distributions (Johnson, Kotz \& Balakrishnan (1994)).

Given $N$, let $X_{1}, X_{2}, \ldots, X_{N}$ be identically and independently distributed (iid) random variable following the Weibull-G distributions. Let $X_{(n)}=\max \left(X_{1}, X_{2}, \ldots, X_{n}\right)$, then the cdf of $X_{(n)} \mid N=n$ is given by

$$
F_{X_{(n)} \mid N=n}(x ; \alpha, \beta, \xi)=\left(1-\exp \left\{-\alpha\left[\frac{G(x ; \xi)}{\bar{G}(x ; \xi)}\right]^{\beta}\right\}\right)^{n}, \alpha, \beta, \xi>0, n \geq 1 .
$$

The Weibull-G Power Series (WGPS) distribution denoted by WGPS $(\alpha, \beta, \theta, \xi)$ is defined by the marginal distribution of $X_{(n)}$, and is given by

$$
\begin{aligned}
F_{X_{(n)}}(x) & =\sum_{n=1}^{\infty} P\left(X_{(n)} \leq x, N=n\right) \\
& =\sum_{n=1}^{\infty} P\left(X_{(n)}<x \mid N=n\right) P(N=n) \\
& =\sum_{n=1}^{\infty} \frac{a_{n} \theta^{n}}{C(\theta)}\left(1-\exp \left\{-\alpha\left[\frac{G(x ; \xi)}{\bar{G}(x ; \xi)}\right]^{\beta}\right\}\right)^{n} \\
F_{X_{(n)}}(x) & =\sum_{n=1}^{\infty} \frac{a_{n}}{C(\theta)}\left(\theta\left(1-\exp \left\{-\alpha\left[\frac{G(x ; \xi)}{\bar{G}(x ; \xi)}\right]^{\beta}\right\}\right)\right)^{n} \\
& =\frac{C\left(\theta\left(1-\exp \left\{-\alpha\left[\frac{G(x ; \xi)}{\bar{G}(x ; \xi}\right]^{\beta}\right\}\right)\right)}{C(\theta)}, x>0 .
\end{aligned}
$$

The pdf is given by

$$
f_{X_{(n)}}(x)=\theta \alpha \beta g(x ; \xi) \frac{(G(x ; \xi))^{\beta-1}}{(\bar{G}(x ; \xi))^{\beta+1}} e^{-\alpha\left[\frac{G(x ; \xi)}{\bar{G}(x ; \xi)}\right]^{\beta}} \frac{C^{\prime}\left(\theta\left(1-\exp \left\{-\alpha\left[\frac{G(x ; \xi)}{\bar{G}(x ; \xi)}\right]^{\beta}\right\}\right)\right)}{C(\theta)} .
$$


The hazard function is given by

$$
h_{F}(x)=\frac{\theta \alpha \beta g(x ; \xi) \frac{(G(x ; \xi))^{\beta-1}}{(\bar{G}(x ; \xi))^{\beta+1}} e^{-\alpha\left[\frac{G(x ; \xi)}{\bar{G}(x ; \xi)}\right]^{\beta}} C^{\prime}\left(\theta\left(1-\exp \left\{-\alpha\left[\frac{G(x ; \xi)}{\bar{G}(x ; \xi}\right]^{\beta}\right\}\right)\right)}{C(\theta)-C\left(\theta\left(1-\exp \left\{-\alpha\left[\frac{G(x ; \xi)}{\bar{G}(x ; \xi)}\right]^{\beta}\right\}\right)\right)} .
$$

\subsection{Quantile Function}

Let $\mathrm{X}$ be a random variable with cdf as in (5). The quantile function $Q_{X_{(n)}}(u)$ is defined by $F_{X_{(n)}}\left(Q_{X_{(n)}}(u)\right)=u, 0 \leq u \leq 1$. Therefore,

$$
Q_{X_{(n)}}(u)=G^{-1}\left(\frac{\left(-\frac{1}{\alpha} \ln \left(1-\frac{C^{-1}(u(C(\theta)))}{\theta}\right)\right)^{1 / \beta}}{1+\left(-\frac{1}{\alpha} \ln \left(1-\frac{C^{-1}(u(C(\theta)))}{\theta}\right)\right)^{1 / \beta}}\right) .
$$

\subsection{Expansion of Density}

The series expansion of the pdf of the WGPS class of distributions is presented in this sub-section. We apply the series expansion $(1-y)^{-k}=\sum_{j=0}^{\infty} \frac{\Gamma(k+j)}{j ! \Gamma(k)} y^{j},|y|<1, k>0$, to rewrite the WGPS density function as

$$
\begin{aligned}
f_{X_{(n)}}(x) & =\theta \alpha \beta g(x ; \xi) \frac{G(x ; \xi)^{\beta-1}}{\bar{G}(x ; \xi)^{\beta+1}} e^{-\alpha\left[\frac{G(x ; \xi)}{\bar{G}(x ;)}\right]^{\beta}} \frac{C^{\prime}\left(\theta\left(1-e^{-\alpha\left[\frac{G(x ; \xi)}{\bar{G}(x ; \xi)}\right]^{\beta}}\right)\right)}{C(\theta)} \\
& =\sum_{n=1}^{\infty} \frac{a_{n} \theta^{n}}{C(\theta)} n \alpha \beta g(x ; \xi) \frac{G(x ; \xi)^{\beta-1}}{\bar{G}(x ; \xi)^{\beta+1}} e^{-\alpha\left[\frac{G(x ; \xi)}{\bar{G}(x ;)}\right]^{\beta}}\left[1-e^{-\alpha\left[\frac{G(x ; \xi)}{\bar{G}(x ; \xi)}\right]^{\beta}}\right]^{n-1} \\
& =\sum_{j=0}^{\infty} \sum_{n=1}^{\infty} \frac{a_{n} \theta^{n}(-1)^{j} n ! \alpha(j+1) \beta}{C(\theta) \Gamma(j)(n-j-1) !} g(x ; \xi) \frac{G(x ; \xi)^{\beta-1}}{\bar{G}(x ; \xi)^{\beta+1}} e^{-\alpha(j+1)\left[\frac{G(x ; \xi)}{\bar{G}\left(x_{i}\right)}\right]^{\beta}} \\
& =\sum_{j=0}^{\infty} \sum_{n=1}^{\infty} \omega_{j, n}(\theta) f_{W G}(x ; \alpha(j+1), \beta, \xi),
\end{aligned}
$$

where

$$
f_{W G}(x ; \theta, \alpha(j+1), \beta, \xi)=\alpha(j+1) \beta g(x ; \xi) \frac{G(x ; \xi)^{\beta-1}}{\bar{G}(x ; \xi)^{\beta+1}} e^{-\alpha(j+1)\left[\frac{G(x ; \xi)}{G(x ; \xi \xi}\right]^{\beta}}
$$

for $\theta>0, \alpha(j+1)>0, \beta>0$ and $\xi>0$, is the Weibull-G (WG) family of distributions and $\omega_{j, n}(\theta)=\left\{(-1)^{j} n ! a_{n} \theta^{n}\right\} /\{C(\theta)(n-$ $j-1) ! \Gamma(j)\}$ are the weights. It follows that the WGPS class of distributions can be written as a linear combination of WG class of distributions. Consequently, the mathematical and statistical properties of the WGPS class of distributions can be readily obtained from those of the WG class of distributions.

\subsection{Moments}

Moments are very crucial in any statistical analysis. In this subsection, we present the $r^{\text {th }}$ moment and moment generating function (MGF) of the WGPS class of distributions. The $r^{\text {th }}$ moment is given by

$$
E\left(X^{r}\right)=\sum_{j=0}^{\infty} \sum_{n=1}^{\infty} \omega_{j, n}(\theta) \int_{-\infty}^{\infty} x^{r} f_{W G}(x ; \alpha(j+1), \beta, \xi) d x=\sum_{j=0}^{\infty} \sum_{n=1}^{\infty} \omega_{j, n}(\theta) E\left(Y^{r}\right),
$$

where $Y \sim W G(\alpha(j+1), \beta, \xi)$. Note that

$$
\begin{aligned}
E\left(Y^{r}\right) & =\int_{-\infty}^{\infty} y^{r} f_{W G}(y ; \alpha(j+1), \beta, \theta) d y \\
& =\alpha(j+1) \beta \int_{-\infty}^{\infty} y^{r} g(y ; \xi) \frac{G(y ; \xi)^{\beta-1}}{\bar{G}(y ; \xi)^{\beta+1}} e^{-\alpha(j+1)\left[\frac{G(5 ; \xi)}{G(j ;)}\right]^{\beta}} d y \\
& =\sum_{k=0}^{\infty} \sum_{m=0}^{\infty} \frac{(-1)^{k} \Gamma(\beta(k+1)+m+1)(\alpha(j+1))^{k+1} \beta}{k ! m ! \Gamma(\beta(k+1)+1)} \int_{-\infty}^{\infty} y^{r} g(y ; \xi) G(y ; \xi)^{\beta(k+1)+m-1} d y .
\end{aligned}
$$

Thus, the moments of any WG distribution can be expressed as an infinite weighted sum of probability weighted moments (PWMs) of the parent distribution. The integral $\int_{-\infty}^{\infty} y^{r} g(y ; \xi) G(y ; \xi)^{\beta(k+1)+m-1} d y$ can be based on the parent quantile 
function $Q_{G}(y)=G^{-1}(y ; \xi)$. Now, by setting $G(y ; \xi)=u$, we obtain

$$
\int_{-\infty}^{\infty} y^{r} G(y ; \xi)^{k} g(y ; \xi) d y=\int_{0}^{1} Q_{G}(u)^{r} u^{k} d u
$$

where the integral is now calculated over the interval $(0,1)$. The MGF of the WGPS class of distributions is given by

$$
E\left(e^{t X}\right)=\sum_{i=0}^{\infty} \frac{t^{i}}{i !} E\left(X^{i}\right),
$$

where $E\left(X^{r}\right)$ is given by the equation (9). Note also that the $r^{\text {th }}$ moment of the WGPS class of distributions can be directly obtained as follows:

$$
\begin{aligned}
E\left(X^{r}\right) & =\sum_{j=0}^{\infty} \sum_{k=0}^{\infty} \sum_{m=0}^{\infty} \sum_{n=1}^{\infty} \omega_{j, n}(\theta) \frac{(-1)^{k} \Gamma(\beta(k+1)+m+1)(\alpha(j+1))^{k+1} \beta}{k ! m ! \Gamma(\beta(k+1)+1)} \int_{-\infty}^{\infty} x^{r} g(x ; \xi) G(x ; \xi)^{\beta(k+1)+m-1} d x \\
& =\sum_{j=0}^{\infty} \sum_{k=0}^{\infty} \sum_{m=0}^{\infty} \sum_{n=1}^{\infty} \omega_{j, n}(\theta) \frac{(-1)^{k} \Gamma(\beta(k+1)+m+1)(\alpha(j+1))^{k+1} \beta}{k ! m ! \Gamma(\beta(k+1)+1)} \int_{0}^{1} Q_{G}(u)^{r} u^{\beta(k+1)+m-1} d u .
\end{aligned}
$$

\subsection{Conditional Moments}

The conditional moments of the WGPS class of distributions is presented in this subsection. They can be used to calculate the mean residual function and other useful quantities in reliability and survival analysis. The $r^{\text {th }}$ conditional moment for the WGPS class of distributions is given by

$$
\begin{aligned}
E\left(X^{r} \mid X>t\right) & =\frac{1}{\bar{F}_{W G P S}(t)} \int_{t}^{\infty} x^{r} f_{W G P S}(x ; \theta, \alpha(j+1), \beta \xi) d x \\
& =\frac{1}{\bar{F}_{W G P S}(t)} \sum_{j=0}^{\infty} \sum_{n=1}^{\infty} \omega_{j, n}(\theta) \int_{t}^{\infty} x^{r} \alpha(j+1) \beta g(x ; \xi) \frac{G(x ; \xi)^{\beta-1}}{\bar{G}(x ; \xi)^{\beta+1}} e^{-\alpha(j+1)\left[\frac{G(x ; \xi)}{G(x ; \xi)}\right]^{\beta}} d x \\
& =\frac{1}{\bar{F}_{W G P S}(t)} \sum_{j=0}^{\infty} \sum_{k=0}^{\infty} \sum_{m=0}^{\infty} \sum_{n=1}^{\infty} \omega_{j, n}(\theta) \frac{(-1)^{k} \Gamma(\beta(k+1)+m+1)}{k ! m ! \Gamma(\beta(k+1)+1)}(\alpha(j+1))^{k+1} \beta \int_{t}^{\infty} x^{r} g(x ; \xi) G(x ; \xi)^{\beta(k+1)+m-1} d x .
\end{aligned}
$$

The WGPS vitality and mean residual life functions are given by $E(Y \mid Y>t)$ and $E(Y \mid Y>t)-t$, respectively.

\subsection{Order Statistics, L-Moments and Rényi Entropy}

This section contain the distribution of the order statistic, L-moments and Rényi entropy of the WGPS class of distributions.

\subsubsection{Order Statistics}

The pdf of the $i^{\text {th }}$ order statistic from the WGPS pdf $f_{W G L}(x)$ is given by

$$
\begin{aligned}
g_{i: n}(x) & =\frac{n ! f_{\text {WGPS }}(x)}{(i-1) !(n-i) !}\left[F_{\text {WGPS }}(x)\right]^{i-1}\left[1-F_{\text {WGPS }}(x)\right]^{n-i} \\
& =\frac{n ! f_{\text {WGPS }}(x)}{(i-1) !(n-i) !} \sum_{j=0}^{n-i}(-1)^{j}\left(\begin{array}{c}
n-i \\
j
\end{array}\right)\left[F_{W G P S}(x)\right]^{j+i-1}
\end{aligned}
$$

by using the binomial expansion $\left[1-F_{\text {WGPS }}(x)\right]^{n-i}=\sum_{m=0}^{n-i}\left(\begin{array}{c}n-i \\ m\end{array}\right)(-1)^{m}\left[F_{\text {WGPS }}(x)\right]^{m}$, so that

$$
\begin{aligned}
g_{i: n}(x) & =\frac{1}{B(i, n-i+1)} \sum_{m=0}^{n-i}\left(\begin{array}{c}
n-i \\
m
\end{array}\right) \frac{(-1)^{m}}{m+i}(m+i)\left[F_{W G P S}(x)\right]^{m+i-1} f_{W G P S}(x) \\
& =\sum_{m=0}^{n-i} w_{i, m} f_{m+i}(x)
\end{aligned}
$$

where $f_{m+i}(x)$ is the pdf of the exponentiated WGPS distribution with parameters $\theta, \alpha, \beta, \xi$ and $(m+i), B(.,$.$) is the beta$ function and the weights $w_{i, m}$ are given by

$$
w_{i, m}=\frac{1}{B(i, n-i+1)} \frac{(-1)^{m}}{m+i}\left(\begin{array}{c}
n-i \\
m
\end{array}\right)=(-1)^{m}\left(\begin{array}{c}
m+i-1 \\
m
\end{array}\right)\left(\begin{array}{c}
n \\
m+i
\end{array}\right) .
$$


The $t^{\text {th }}$ moment of the $i^{\text {th }}$ order statistics from the WGPS distribution can be obtained by using the result given by Barakat \& Abdelkhader (2004) as follows:

$$
E\left(X_{i: n}^{t}\right)=t \sum_{p=n-i+1}^{n}(-1)^{p-n+i-1}\left(\begin{array}{l}
p-1 \\
n-i
\end{array}\right)\left(\begin{array}{l}
n \\
p
\end{array}\right) \int_{0}^{\infty} x^{t-1}\left[1-F_{\text {WGPS }}(x)\right]^{p} d x
$$

Note that

$$
\begin{aligned}
\int_{0}^{\infty} x^{t-1}\left[\bar{F}_{\text {WGPS }}(x)\right]^{p} d x & =\int_{0}^{\infty} x^{t-1}\left[1-\frac{\left.C\left(\theta\left(1-\exp \left\{-\alpha\left[\frac{G(x ; \xi)}{\bar{G}(x ; \xi)}\right]^{\beta}\right\}\right)\right)\right]^{p}}{C(\theta)} d x\right. \\
& =\sum_{k=0}^{\infty}(-1)^{k}\left(\begin{array}{l}
p \\
k
\end{array}\right) \int_{0}^{\infty} x^{t-1}\left(\frac{C\left(\theta\left(1-\exp \left\{-\alpha\left[\frac{G(x ; \xi)}{\bar{G}(x ; \xi)}\right]^{\beta}\right\}\right)\right)}{C(\theta)}\right)^{k} d x \\
& =\sum_{k=0}^{\infty}(-1)^{k}\left(\begin{array}{l}
p \\
k
\end{array}\right) \int_{0}^{\infty} x^{t-1}\left(\sum_{s=0}^{\infty} \frac{a_{s+1} \theta^{s+1}\left(1-\exp \left\{-\alpha\left[\frac{G(x ; \xi)}{\bar{G}(x ; \xi)}\right]^{\beta}\right\}\right)^{s+1}}{C(\theta)}\right)^{k} d x .
\end{aligned}
$$

Now, applying the result on power series raised to a positive integer from (Gradshetyn \& Ryzhik (2000)), with $a_{s}=a_{s+1} \theta^{s}$, that is,

$$
\left(\sum_{s=0}^{\infty} a_{s} y^{s}\right)^{k}=\sum_{s=0}^{\infty} b_{s, k} y^{s}
$$

where $b_{s, k}=\left(s a_{0}\right)^{-1} \sum_{l=1}^{s}[k(l+1)-s] a_{l} b_{s-l, k}$, and $b_{0, k}=a_{0}^{k}$, we obtain

$$
\begin{aligned}
& \int_{0}^{\infty} x^{t-1}\left[\bar{F}_{W G P S}(x)\right]^{p} d x=\sum_{k=0}^{\infty} \frac{(-1)^{k} \theta^{k}}{C(\theta)^{k}}\left(\begin{array}{l}
p \\
k
\end{array}\right) \int_{0}^{\infty} x^{t-1}\left(1-e^{-\alpha\left[\frac{G(x ; \xi)}{G(x ; x)}\right]^{\beta}}\right)^{k}\left(\sum_{s=0}^{\infty} a_{s+1} \theta^{s}\left(1-e^{-\alpha\left[\frac{G(x ; \xi)}{G(x, \xi)}\right]^{\beta}}\right)^{s}\right)^{k} d x \\
& =\sum_{k=0}^{\infty} \frac{(-1)^{k} \theta^{k}}{C(\theta)^{k}}\left(\begin{array}{l}
p \\
k
\end{array}\right) \int_{0}^{\infty} x^{t-1}\left(1-e^{-\alpha\left[\frac{G(x ; \xi \xi}{G(x ; k)}\right]^{\beta}}\right)^{k} \sum_{s=0}^{\infty} b_{s, k}\left(1-e^{-\alpha\left[\frac{G(x ; z)}{G(x ; k)}\right]^{\beta}}\right)^{s} d x \\
& =\sum_{k=0}^{\infty} \sum_{s=0}^{\infty} b_{s, k} \frac{(-1)^{k} \theta^{k}}{C(\theta)^{k}}\left(\begin{array}{l}
p \\
k
\end{array}\right) \int_{0}^{\infty} x^{t-1}\left(1-e^{-\alpha\left[\frac{G(x, \xi)}{G(x, \xi)}\right]^{\beta}}\right)^{k+s} d x \\
& =\sum_{k=0}^{\infty} \sum_{s, w=0}^{\infty} b_{s, k} \frac{(-1)^{k+w} \theta^{k}}{C(\theta)^{k}}\left(\begin{array}{l}
p \\
k
\end{array}\right)\left(\begin{array}{c}
k+s \\
w
\end{array}\right) \int_{0}^{\infty} x^{t-1} e^{-\alpha w\left[\frac{G(x ; \xi)}{G(x ; \xi)}\right]^{\beta}} d x .
\end{aligned}
$$

\subsubsection{L-Moments}

The $L$-moments (Hoskings (1990)) are expectations of some linear combinations of order statistics and they exist whenever the mean of the distribution exits, even when some higher moments may not exist. They are given by

$$
\lambda_{k+1}=\frac{1}{k+1} \sum_{j=0}^{k}(-1)^{j}\left(\begin{array}{l}
k \\
j
\end{array}\right) E\left(X_{k+1-j: k+1}\right), \quad k=0,1,2, \ldots \ldots .
$$

The $L$-moments of the WGPS distribution are obtained from equation (14). The first four $L$-moments are given by $\lambda_{1}=E\left(X_{1: 1}\right), \lambda_{2}=\frac{1}{2} E\left(X_{2: 2}-X_{1: 2}\right), \lambda_{3}=\frac{1}{3} E\left(X_{3: 3}-2 X_{2: 3}+X_{1: 3}\right)$ and $\lambda_{4}=\frac{1}{4} E\left(X_{4: 4}-3 X_{3: 4}+3 X_{2: 4}-X_{1: 4}\right)$, respectively.

\subsubsection{Rényi Entropy}

Rényi entropy is an extension of Shannon entropy and is given by

$$
I_{R}(v)=\frac{1}{1-v} \log \left(\int_{0}^{\infty}\left[f_{W G P S}(x ; \theta, \alpha, \beta, \xi)\right]^{v} d x\right), v \neq 1, v>0 .
$$


Rényi entropy tends to Shannon entropy as $v \rightarrow 1$. Note that $\left[f_{W G P S}(x ; \theta, \alpha, \beta, \xi)\right]^{v}=f_{W G P S}^{v}(x)$ can be written as

$$
\begin{aligned}
f_{W G P S}^{v}(x) & =\left[\frac{\theta \alpha \beta g(x ; \xi)}{C(\theta)} \frac{(G(x ; \xi))^{\beta-1}}{(\bar{G}(x ; \xi))^{\beta+1}} e^{-\alpha\left[\frac{G(x ; \xi)}{\bar{G}(x ; \xi)}\right]^{\beta}} C^{\prime}\left(\theta\left(1-e^{-\alpha\left[\frac{G(x ; \xi)}{\bar{G}(x ; \xi)}\right]^{\beta}}\right)\right)\right]^{v} \\
& =\sum_{s=0}^{\infty} \sum_{j=0}^{\infty} b_{s, k}\left(\begin{array}{l}
v \\
j
\end{array}\right) \frac{(-1)^{j}(\theta \alpha \beta g(x ; \xi))^{v}}{C(\theta)^{v}} \frac{(G(x ; \xi))^{(\beta-1) v}}{(\bar{G}(x ; \xi))^{(\beta+1) v}} e^{-\alpha(v+j)\left[\frac{G(x ; \xi)}{\bar{G}(x ; \xi)}\right]^{\beta}} \\
& =\sum_{s=0}^{\infty} \sum_{j, k, l=0}^{\infty} b_{s, k}(-1)^{j+k}\left(\begin{array}{l}
v \\
j
\end{array}\right) \frac{(\alpha(v+j))^{k}}{k !} \frac{\Gamma(v(\beta+1)+\beta k+l)}{l ! \Gamma(v(\beta+k)+\beta k)} \frac{(\theta \alpha \beta g(x ; \xi))^{v}}{C(\theta)^{v}} G(x ; \xi)^{v(\beta-1)+\beta k+l} .
\end{aligned}
$$

Rényi entropy for the WGPS class of distributions is given by

$$
\begin{aligned}
I_{R}(v) & =\frac{1}{1-v} \log \left[\sum_{s=0}^{\infty} \sum_{j, k, l=0}^{\infty} b_{s, k}(-1)^{j+k}\left(\begin{array}{l}
v \\
j
\end{array}\right) \frac{(\alpha(v+j))^{k}}{k !} \frac{\Gamma(v(\beta+1)+\beta k+l)}{l ! \Gamma(v(\beta+k)+\beta k)}\right. \\
& \left.\times \frac{(\theta \alpha \beta)^{v}}{C(\theta)^{v}} \int_{0}^{\infty} g(x ; \xi)^{v} G(x ; \xi)^{v(\beta-1)+\beta k+l} d x\right],
\end{aligned}
$$

for $v \neq 1$, and $v>0$.

\subsection{Maximum Likelihood Estimation}

Let $X_{i} \sim W G P S(\alpha, \beta, \theta, \xi)$ and $\Delta=(\alpha, \beta, \theta, \xi)^{T}$ be the parameter vector. The log-likelihood $\ell=\ell(\Delta)$ based on a random sample of size $\mathrm{n}$ is given by

$$
\begin{aligned}
\ell & =\ell(\Delta)=n \ln [\alpha \beta \theta]-n \ln [C(\theta)]+\sum_{i=1}^{n} \ln \left[g\left(x_{i} ; \xi\right)\right]+(\beta-1) \sum_{i=1}^{n} \ln \left[G\left(x_{i} ; \xi\right)\right] \\
& -(\beta+1) \sum_{i=1}^{n} \ln \left[\bar{G}\left(x_{i} ; \xi\right)\right]-\alpha \sum_{i=1}^{n} H\left(x_{i} ; \xi\right)^{\beta}+\sum_{i=1}^{n} \ln \left[C^{\prime}\left(\theta\left(1-e^{\left(-\alpha H\left(x_{i} ; \xi\right)\right)^{\beta}}\right)\right)\right],
\end{aligned}
$$

where $H\left(x_{i} ; \xi\right)=G\left(x_{i} ; \xi\right) / \bar{G}\left(x_{i} ; \xi\right)$, and $\bar{G}\left(x_{i} ; \xi\right)=1-G\left(x_{i} ; \xi\right)$. Elements of the score vector $U=\left(\frac{\partial \ell}{\partial \alpha}, \frac{\partial \ell}{\partial \beta}, \frac{\partial \ell}{\partial \theta}, \frac{\partial \ell}{\partial \xi_{k}}\right)$ are given by:

$$
\begin{gathered}
\frac{\partial \ell}{\partial \alpha}=\frac{n}{\alpha}-\sum_{i=1}^{n} H\left(x_{i} ; \xi\right)^{\beta}+\theta \sum_{i=1}^{n} H\left(x_{i} ; \xi\right)^{\beta} e^{-\alpha H\left(x_{i} ; \xi^{\beta}\right.} \frac{C^{\prime \prime}\left(\theta\left(1-e^{-\alpha H\left(x_{i} ; \xi^{\beta}\right.}\right)\right)}{C^{\prime}\left(\theta\left(1-e^{-\alpha H\left(x_{i} ; \xi^{\beta}\right.}\right)\right)} \\
\frac{\partial \ell}{\partial \beta}=\frac{n}{\beta}+\sum_{i=1}^{n} H\left(x_{i} ; \xi\right)-\sum_{i=1}^{n} H\left(x_{i} ; \xi\right)^{\beta} \ln \left(H\left(x_{i} ; \xi\right)\right)+\sum_{i=1}^{n} H\left(x_{i} ; \xi\right)^{\beta} e^{-\alpha H\left(x_{i} ; \xi\right)^{\beta}} \ln \left(H\left(x_{i} ; \xi\right)\right) \frac{C^{\prime \prime}\left(\theta\left(1-e^{-\alpha H\left(x_{i} ; \xi\right)^{\beta}}\right)\right)}{C^{\prime}\left(\theta\left(1-e^{-\alpha H\left(x_{i} ; \xi\right)^{\beta}}\right)\right)}, \\
\frac{\partial \ell}{\partial \theta}=\frac{n}{\theta}-\frac{n C^{\prime}(\theta)}{C(\theta)}+\sum_{i=1}^{n}\left(1-e^{-\alpha H\left(x_{i} ; \xi\right)^{\beta}}\right) \frac{C^{\prime \prime}\left(\theta\left(1-e^{-\alpha H\left(x_{i} ; \xi^{\beta}\right.}\right)\right)}{C^{\prime}\left(\theta\left(1-e^{-\alpha H\left(x_{i} ; \xi^{\beta}\right.}\right)\right)}
\end{gathered}
$$

and

$$
\begin{aligned}
\frac{\partial \ell}{\partial \xi_{k}} & =\sum_{i=1}^{n} \frac{\partial g\left(x_{i} ; \xi\right) / \partial \xi_{k}}{g\left(x_{i} ; \xi\right)}+(\beta-1) \sum_{i=1}^{n} \frac{\partial G\left(x_{i} ; \xi\right) / \partial \xi_{k}}{G\left(x_{i} ; \xi\right)}-(\beta+1) \sum_{i=1}^{n} \frac{\partial \bar{G}\left(x_{i} ; \xi\right) / \partial \xi_{k}}{\bar{G}\left(x_{i} ; \xi\right)}-\alpha \beta \sum_{i=1}^{n} H\left(x_{i} ; \xi\right)^{\beta-1} \frac{\partial H\left(x_{i} ; \xi\right)}{\partial \xi_{k}} \\
& +\alpha \beta \theta \sum_{i=1}^{n} H\left(x_{i} ; \xi\right)^{\beta-1} e^{-\alpha H\left(x_{i} ; \xi^{\beta}\right.} \frac{\partial H\left(x_{i} ; \xi\right)}{\partial \xi_{k}} \frac{C^{\prime \prime}\left(\theta\left(1-e^{-\alpha H\left(x_{i} ; \xi^{\beta}\right.}\right)\right)}{C^{\prime}\left(\theta\left(1-e^{-\alpha H\left(x_{i} ; \xi^{\beta}\right.}\right)\right)}
\end{aligned}
$$

respectively. The solution of the non-linear system equations, $U=\left(\frac{\partial \ell}{\partial \alpha}, \frac{\partial \ell}{\partial \beta}, \frac{\partial \ell}{\partial \theta}, \frac{\partial \ell}{\partial \xi_{k}}\right)=\mathbf{0}$ gives the maximum likelihood estimates. These equations can be solved numerically via an iterative methods such as Newton-Raphson technique using statistical software.

\section{The Sub-model of WGPS Class of Distributions and Properties}

In this section, we derive some properties of the Weibull-G logarithmic (WGL) family of distributions. 
Let the random variable $X$ denote the lifetime of a system defined by $X=\max \left(X_{1}, X_{2}, \ldots, X_{N}\right)$, where we assume that the distribution of each $X_{i}$ is identically and independently distributed following the Weibull-G family of distributions with cdf and pdf given in equations (1) and (2). Let the random variable $N$ follow the logarithmic distribution with pmf given as:

$$
P(N=n)=\left[\frac{p^{n}}{-n \ln (1-p)}\right], \quad n=1,2, \ldots, \text { and } 0<p<1 .
$$

Then the marginal cdf and pdf of $X$ are given by

$$
\begin{aligned}
F_{W G L}(x) & =\sum_{n=1}^{\infty} P(X \leq x \mid N=n) P(N=n) \\
& =\frac{\ln \left[1-p\left(1-\exp \left\{-\alpha\left[\frac{G(x ; \xi)}{\bar{G}(x ; \xi)}\right]^{\beta}\right\}\right)\right]}{\ln (1-p)},
\end{aligned}
$$

and

$$
f_{W G L}(x ; p, \alpha, \beta, \xi)=\frac{p \alpha \beta g(x ; \xi)\left[\frac{G(x ; \xi)^{\beta-1}}{\bar{G}\left(x ; \xi^{\beta+1}\right.}\right] \exp \left\{-\alpha\left[\frac{G(x ; \xi)}{\bar{G}(x ; \xi)}\right]^{\beta}\right\}}{\ln (1-p)\left[p\left(1-\exp \left\{-\alpha\left[\frac{G(x ; \xi)}{\bar{G}(x ; \xi)}\right]^{\beta}\right\}\right)-1\right]}
$$

for $\alpha>0,0<p<1, \beta>0$, respectively.

\subsection{Quantile Function}

The WGL quantile function can be obtained by inverting $F_{W G L}(x)=u, 0 \leq u \leq 1$, where

$$
F_{W G L}(x)=\frac{\ln \left(1-p\left(1-\exp \left\{-\alpha\left[\frac{G(x ; \xi)}{\bar{G}(x ; \xi)}\right]^{\beta}\right\}\right)\right)}{\ln (1-p)} .
$$

Note that the quantile function of the WGL distribution is obtained by solving the nonlinear equation

$$
1-e^{-\alpha\left[\frac{G(x ;)}{G(x ; \xi)}\right]^{\beta}}=\frac{1-(1-p)^{u}}{p},
$$

that is,

$$
G(x, \xi)=\frac{\ln \left[\frac{1}{\left(1-\frac{1-(1-p)^{u}}{p}\right)^{\frac{1}{\alpha}}}\right]^{\frac{1}{\beta}}}{1+\ln \left[\frac{1}{\left(1-\frac{1-(1-p)^{u}}{p}\right)^{\frac{1}{\alpha}}}\right]^{\frac{1}{\beta}}} .
$$

Thus, the quantile function of the WGL distribution reduces to

$$
x=G^{-1}\left(\frac{\left[-\frac{1}{\alpha} \ln \left(1-\frac{1-(1-p)^{u}}{p}\right)\right]^{\frac{1}{\beta}}}{1+\left[-\frac{1}{\alpha} \ln \left(1-\frac{1-(1-p)^{u}}{p}\right)\right]^{\frac{1}{\beta}}}\right) .
$$

Consequently, random numbers can be generated based on equation (21), when the function $G(x)$ is specified. Consider a special case where $G(x)$ is Log-logistic (LLo) distribution. Table 1 lists the quantile for selected values of the parameters of the WLLoL distribution.

Table 1. Table of Quantiles for Selected Parameters Values of the WLLoL Distribution

\begin{tabular}{c|ccccc}
\hline $\mathrm{u}$ & $(3,0.1,1.5,0.3)$ & $(1,0.1,2,0.3)$ & $(2,0.1,0.8,0.5)$ & $(4,0.1,0.5,0.5)$ & $(5,0.2,2,0.9)$ \\
\hline 0.1 & 1.049359 & 1.114443 & 1.254837 & 1.199119 & 1.026365 \\
0.2 & 1.237289 & 1.614678 & 1.985430 & 1.730951 & 1.101898 \\
0.3 & 1.370416 & 2.031935 & 2.633435 & 2.169784 & 1.149838 \\
0.4 & 1.481038 & 2.419820 & 3.259260 & 2.573317 & 1.186392 \\
0.5 & 1.581313 & 2.804050 & 3.896945 & 2.968794 & 1.217056 \\
0.6 & 1.678291 & 3.205839 & 4.579280 & 3.377829 & 1.244595 \\
0.7 & 1.778311 & 3.651892 & 5.352975 & 3.827157 & 1.271097 \\
0.8 & 1.890735 & 4.191883 & 6.310348 & 4.365569 & 1.298883 \\
0.9 & 2.039025 & 4.968242 & 7.725203 & 5.132481 & 1.333218 \\
\hline
\end{tabular}




\subsection{Expansion of Density}

Expansion of the density of the WGL distribution is presented in this sub-section. We apply the series expansion $(1-y)^{-k}=$ $\sum_{j=0}^{\infty} \frac{\Gamma(k+j)}{j ! \Gamma(k)} y^{j},|y|<1, k>0$, to rewrite the WGL density function as follows:

$$
\begin{aligned}
& f_{W G L}(x)=\frac{p \alpha \beta g(x ; \xi)\left[\frac{G\left(x ; \xi^{\beta-1}\right.}{\bar{G}(x ; \xi)^{\beta+1}}\right] \exp \left\{-\alpha\left[\frac{G(x ; \xi)}{\bar{G}(x ; \xi)}\right]^{\beta}\right\}}{\left[p\left(1-\exp \left\{-\alpha\left[\frac{G(x ; \xi)}{\bar{G}(x ; \xi)}\right]^{\beta}\right\}\right)-1\right] \ln (1-p)} \\
& =\frac{-\alpha \beta}{\ln (1-p)} \sum_{i=0}^{\infty} p^{i+1} g(x ; \xi)\left[\frac{G(x ; \xi)^{\beta-1}}{\bar{G}(x ; \xi)^{\beta+1}}\right] e^{-\alpha\left[\frac{G(x ; \xi)}{\bar{G}(x ; \xi)}\right]^{\beta}}\left[1-e^{\left.-\alpha\left[\frac{G(x ; \xi)}{\bar{G}(x ; \xi)}\right]^{\beta}\right]^{i}}\right. \\
& =\sum_{i=0}^{\infty} \sum_{j=0}^{\infty} \frac{(-1)^{j+1} p^{i+1} \Gamma(i+1)}{\ln (1-p) \Gamma(j+2) \Gamma(i-j+1)} \alpha \beta(j+1) g(x ; \xi)\left[\frac{G(x ; \xi)^{\beta-1}}{\bar{G}(x ; \xi)^{\beta+1}}\right] e^{-\alpha(j+1)\left[\frac{G(x ; \xi)}{G(x ; \xi)}\right]^{\beta}} \\
& =\sum_{j=0}^{\infty} \sum_{i=0}^{\infty} \omega_{i, j}(p) f_{W G}(x ; \alpha(j+1), \beta, \xi),
\end{aligned}
$$

where

$$
f_{W G}(x ; \alpha(j+1), \beta, \xi)=\alpha(j+1) \beta g(x ; \xi)\left[\frac{G(x ; \xi)^{\beta-1}}{\bar{G}(x ; \xi)^{\beta+1}}\right] e^{-\alpha(j+1)\left[\frac{G(x ; \xi)}{G(x ; \xi)}\right]^{\beta}}
$$

for $\alpha(j+1)>0, \beta>0, \xi>\underline{0}$, is the Weibull-G (WG) family of distributions and $\omega_{i, j}(p)=\left\{(-1)^{j+1} p^{i+1} \Gamma(i+1)\right\} /\{\ln (1-$ $p) \Gamma(j+2) \Gamma(i-j+1)\}$ are the weights. It follows that the WGL class of distributions can be written as a linear combination of WG class of distributions.

\subsection{Hazard Function}

The hazard function of the WGL distribution is given by

$$
h_{F}(x)=\frac{p \alpha \beta g(x ; \xi)\left[\frac{G(x ; \xi)^{\beta-1}}{\bar{G}\left(x ; \xi^{\beta+1}\right.}\right] \exp \left\{-\alpha\left[\frac{G(x ; \xi)}{\bar{G}(x ; \xi)}\right]^{\beta}\right\}}{\left[p\left(1-e^{-\alpha\left[\frac{G(x ; \xi)}{G(x ; \xi)}\right]^{\beta}}\right)-1\right]\left[\ln (1-p)-\ln \left(1-p\left(1-e^{-\alpha\left[\frac{G(x ; \xi)}{G(x ; \xi)}\right]^{\beta}}\right)\right)\right]}
$$

for $p, \alpha>0, \beta>0$, and the parameter vector $\xi$. Similarly, the reverse hazard function is given by:

$$
\begin{aligned}
\tau_{F}(x) & =\frac{f_{W G L}(x)}{F_{W G L}(x)} \\
& =\frac{p \alpha \beta g(x ; \xi)\left[\frac{G(x ; \xi)^{\beta-1}}{\bar{G}(x ; \xi)^{\beta+1}}\right] \exp \left\{-\alpha\left[\frac{G(x ; \xi)}{\bar{G}(x ; \xi)}\right]^{\beta}\right\}}{\left[p\left(1-e^{-\alpha\left[\frac{G(x ; \xi)}{G(x ; \xi)}\right]^{\beta}}\right)-1\right] \ln \left[1-p\left(1-e^{-\alpha\left[\frac{G(x ; \xi)}{G(x ; \xi)}\right.}\right)\right]}
\end{aligned}
$$

for $p, \alpha>0, \beta>0$, and the parameter vector $\xi$. Plots of the hazard function can be readily obtained for any specified baseline cdf $G(x ; \xi)$.

\subsection{Sub-models of WGL Distribution}

Some special models of the WGL distributions are presented in this subsection. Given the baseline cdf $G(x ; \xi)$, and pdf $g(x ; \xi)$, we present the cdf, pdf and plots of the hazard functions of the Weibull exponential logarithmic, Weibull-Weibull logarithmic, Weibull log-logistic logarithmic, Weibull uniform logarithmic and Weibull Burr XII logarithmic distributions. 
Table 2. Distributions and corresponding $G(x ; \xi) / \bar{G}(x ; \xi)$ functions

\begin{tabular}{llll}
\hline Distribution & $G(x ; \xi) / \bar{G}(x ; \xi)$ & $\Psi=(\alpha, \beta, p, \xi)$ & W-G Logarithmic \\
\hline Exponential $(x>0)$ & $e^{\lambda x}-1$ & $(\alpha, \beta, p, \lambda)$ & W-Exponential L \\
Logistic $(x>0)$ & $e^{\lambda x}$ & $(\alpha, \beta, p, \lambda)$ & W-Logistic L \\
Uniform $(0<x<\theta)$ & $x /(\theta-x)$ & $(\alpha, \beta, p, \theta)$ & W-Uniform L \\
Weibull $(x>0)$ & $e^{\lambda x^{\gamma}}-1$ & $(\alpha, \beta, p, \lambda, \gamma)$ & W-Weibull L \\
Fréchet $(x>0)$ & $\left(e^{\lambda x^{\gamma}}-1\right)^{-1}$ & $(\alpha, \beta, p, \lambda, \gamma)$ & Weibull-Fréchet L \\
Half-logistic $(x>0)$ & $\left(e^{x}-1\right) / 2$ & $(\alpha, \beta, p)$ & W-Half-logistic L \\
Power function $(0<x<1 / \theta)$ & {$\left[(\theta x)^{-k}-1\right]^{-1}$} & $(\alpha, \beta, p, \theta, k)$ & W-Power function L \\
Pareto $(x \geq 0)$ & $(x / \theta)^{k}-1$ & $(\alpha, \beta, p, \theta, k)$ & W-Pareto L \\
Burr XII $(x>0)$ & $\left(1+x^{c}\right)^{k}-1$ & $(\alpha, \beta, p, k, c)$ & W-Burr XII L \\
Log-logistic $(x>0)$ & $x^{c}$ & $(\alpha, \beta, p, c)$ & W-Log-logistic L \\
Lomax $(x>0)$ & $(1+x)^{k}-1$ & $(\alpha, \beta, p, k)$ & W-Lomax L \\
Kumaraswamy $(0<x<1)$ & $\left(1-x^{a}\right)^{-b}-1$ & $(\alpha, \beta, p, a, b)$ & W-Kumaraswamy L \\
Gumbel $(-\infty<x<\infty)$ & {$[\exp (\exp \{-(x-\mu) / \sigma\})-1]^{-1}$} & $(\alpha, \beta, p, \mu, \sigma)$ & W-Gumbel L \\
Normal $(-\infty<x<\infty)$ & $\Phi((x-\mu) / \sigma) /(1-\Phi((x-\mu) / \sigma))$ & $(\alpha, \beta, p, \mu, \sigma)$ & W-Normal L \\
Modified Exponential $(x>0)$ & $e^{\theta x e^{x x}-1}$ & $(\alpha, \beta, p, \theta, \lambda)$ & W-Modified Exponential L \\
\hline
\end{tabular}

\subsubsection{Weibull Exponential Logarithmic Distribution}

The cdf of the exponential distribution for $x>0, \lambda>0$, is $G(x)=1-e^{-\lambda x}$ and pdf given as $g(x)=\lambda e^{-\lambda x}$. Then, we define the Weibull exponential logarithmic (WEL) cdf and the corresponding pdf by

$$
F_{W E L}(x)=\frac{\ln \left[1-p\left[1-e^{-\alpha\left(e^{\lambda x}-1\right)^{\beta}}\right]\right]}{\ln (1-p)} \quad \text { and } \quad f_{W E L}(x)=\frac{p \alpha \beta \lambda\left[e^{\lambda x}-1\right]^{\beta-1} e^{\lambda x-\alpha\left(e^{\lambda x}-1\right)^{\beta}}}{\left[p\left(1-e^{-\alpha\left(e^{\lambda x}-1\right)^{\beta}}\right)-1\right] \ln (1-p)},
$$

respectively, for $\alpha>0, \beta>0, \lambda>0$ and $0<p<1$.

The plots of the WEL density and hazard function for selected parameter values are given in Figure (1a) and (1b), respectively. The density and hazard functions reveal different shapes for various values of the parameters, as shown in these plots. The graph of the hazard function exhibit increasing, decreasing and upside down bathtub shapes for selected values of the parameters. This flexibility makes the WEL hazard function suitable for non-monotonic empirical hazard behavior.

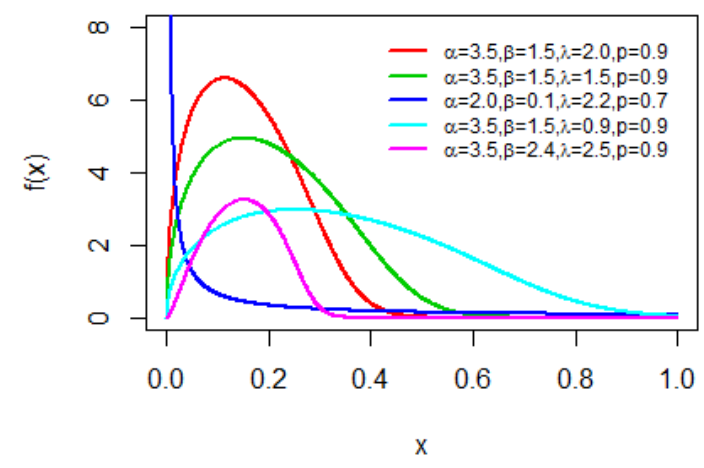

(a) Plot of WEL density function

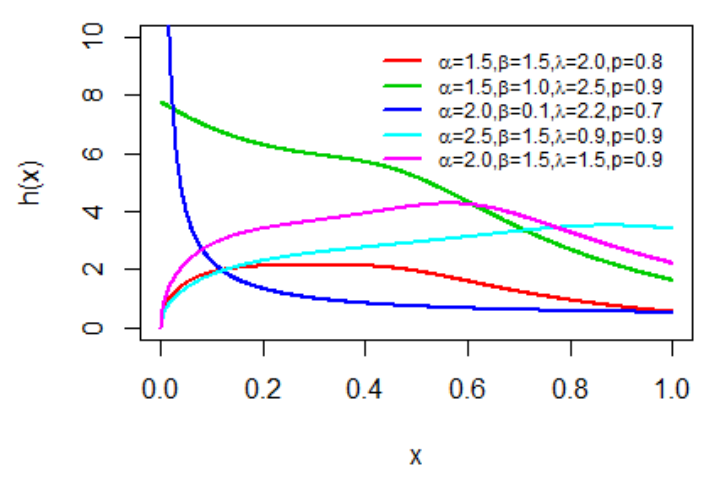

(b) Plot of WEL hazard function

Figure 1. Plots of WEL Density and Hazard Function 


\subsubsection{Weibull-Weibull Logarithmic Distribution}

We define the Weibull-Weibull logarithmic (WWL) cdf and corresponding pdf by

$$
F_{W W L}(x)=\frac{\ln \left[1-p\left[1-e^{-\alpha\left(e^{\lambda x^{\gamma}}-1\right)^{\beta}}\right]\right]}{\ln (1-p)}, \quad \text { and } \quad f_{W W L}(x)=\frac{p \alpha \beta \gamma \lambda x^{\gamma-1}\left[e^{\lambda x^{\gamma}}-1\right]^{\beta-1}\left[e^{\left(\lambda x^{\gamma}-\alpha\left(e^{\lambda x^{\gamma}}-1\right)^{\beta}\right)}\right]}{\left[p\left(1-e^{-\alpha\left(e^{\lambda x^{\gamma}}-1\right)^{\beta}}\right)-1\right] \ln (1-p)},
$$

respectively, for $\alpha>0, \beta>0, \gamma>0, \lambda>0$ and $0<p<1$.

Plots of the WWL density and hazard function for the selected parameter values are given in Figure (2a) and (2b), respectively. The density and hazard functions reveal different shapes for various values of the parameters, as shown in these plots. The graph of the hazard function exhibit various shapes including: increasing, decreasing, bathtub and J-shape for selected values of the parameters.

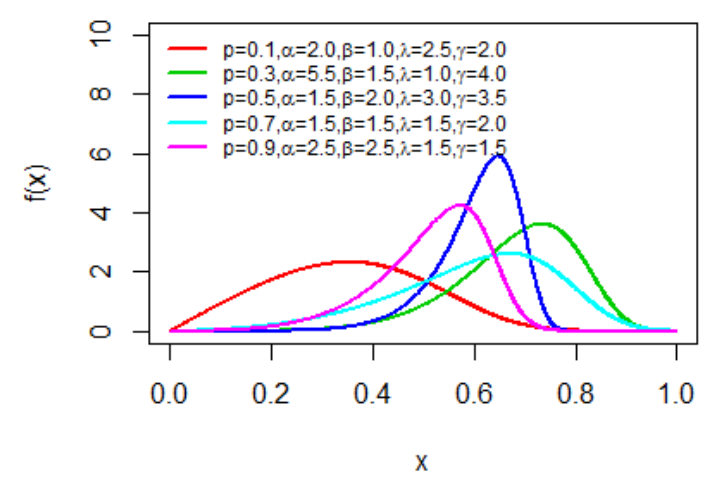

(a) Plot of WWL density function

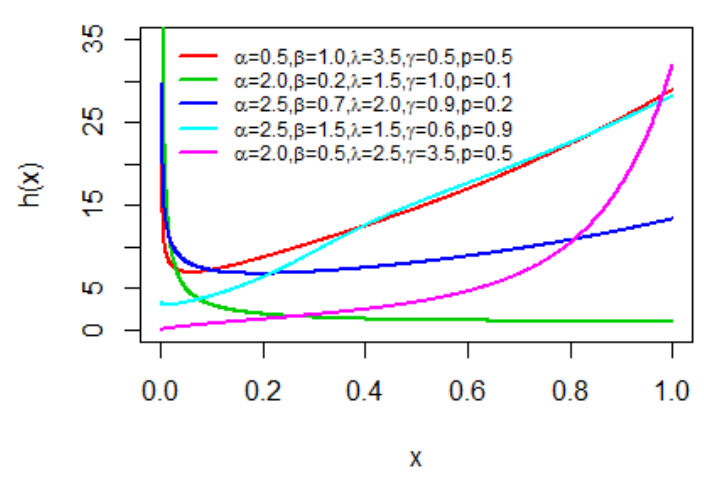

(b) Plot of WWL hazard function

Figure 2. Plots of WWL Density and Hazard Function

\subsubsection{Weibull Log-Logistic Logarithmic Distribution}

The cdf of the log-logistic distribution for $x>0, c>0$, is $G(x)=1-\left(1+x^{c}\right)^{-1}$ and pdf is given as $g(x)=c x^{c-1}\left(1+x^{c}\right)^{-2}$. We define the Weibull log-logistic logarithmic (WLLoL) cdf and the corresponding pdf by

$$
F_{W L L o L}(x)=\frac{\ln \left[1-p\left(1-e^{-\alpha\left(x^{c}\right)^{\beta}}\right)\right]}{\ln (1-p)} \quad \text { and } \quad f_{W L L o L}(x)=\frac{p \alpha \beta c x^{c \beta-1} e^{-\alpha x^{c \beta}}}{\left[p\left(1-e^{-\alpha x^{c \beta}}\right)-1\right] \ln (1-p)},
$$

respectively, for $\alpha>0, \beta>0, c>0$ and $0<p<1$.

Plots of the WLLoL density and hazard function for selected parameter values are given in Figure (3a) and (3b), respectively. The graph of the hazard function exhibit increasing and decreasing shapes for selected values of the parameters, while the density function reveals a decreasing, left skewed and right skewed shapes for various selected values of the parameters, which may also be suitable for analysing extreme values. 


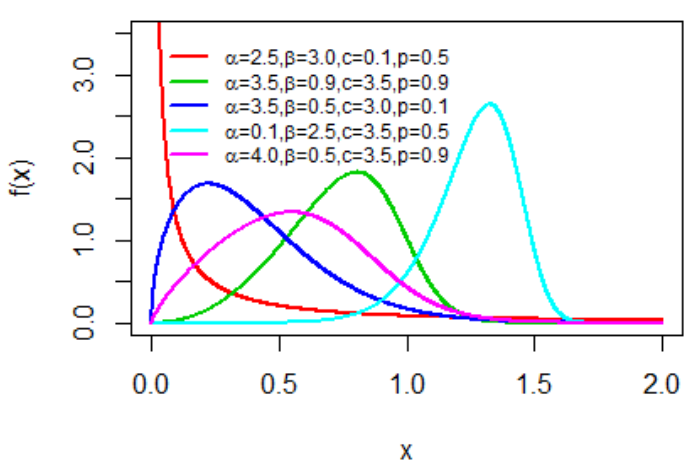

(a) Plot of WLLoL density function

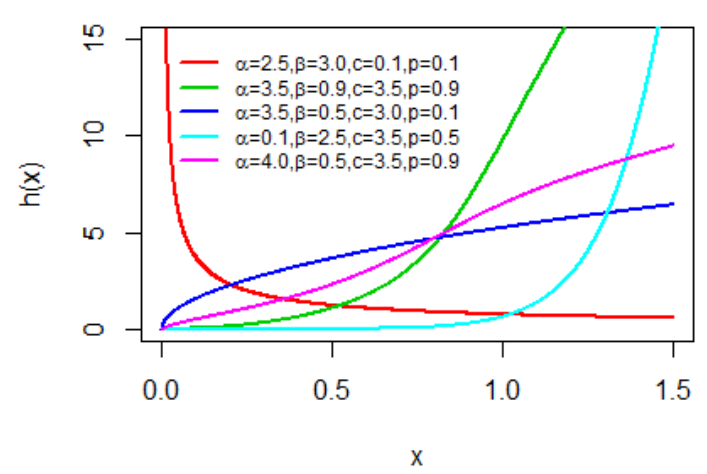

(b) Plot of WLLoL hazard function

Figure 3. Plots of WLLoL Density and Hazard Function

\subsubsection{Weibull Uniform Logarithmic Distribution}

The cdf of the Uniform distribution is $G(x)=\frac{x}{\theta}$ and pdf given as $g(x)=\frac{1}{\theta}$ for $0<x<\theta$. Then we define the Weibull uniform logarithmic (WUL) cdf by

$$
F_{W U L}(x)=\frac{\ln \left[1-p\left(1-e^{-\alpha\left[\frac{x}{\theta-x}\right]^{\beta}}\right)\right]}{\ln (1-p)} \quad \text { and } \quad f_{W U L}(x)=\frac{p \alpha \beta \theta x^{\beta-1} e^{-\alpha\left[\frac{x}{\theta-x}\right]^{\beta}}}{(\theta-x)^{\beta+1}\left[p\left[1-e^{-\alpha\left[\frac{x}{\theta-x}\right]^{\beta}}\right]-1\right] \ln (1-p)},
$$

respectively, for $\alpha>0, \beta>0,0<p<1$ and $0<x<\theta$.

The plots of the WUL density and hazard function for selected parameter values are given in Figure (4a) and (4b) respectively. The density and hazard functions revealed different shapes for various values of the parameters, as shown in these plots. The graph of the hazard function exhibit increasing, decreasing and bathtub shapes for the selected values of the parameters while the density function shows decreasing, left and right skewed shapes for selected values of the parameters.

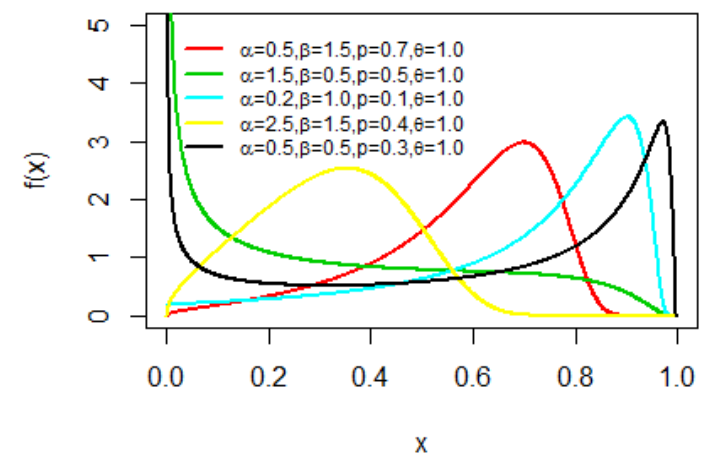

(a) Plot of WUL density function

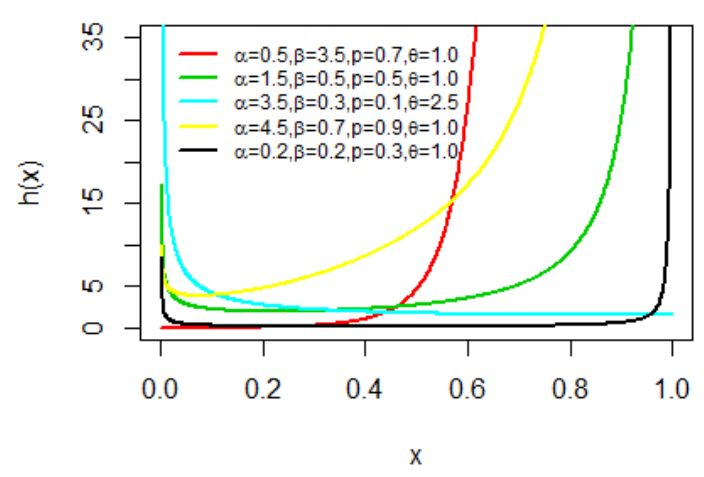

(b) Plot of WUL hazard function

Figure 4. Plots of WUL Density and Hazard Function

\subsubsection{Weibull Burr XII Logarithmic Distribution}

The cdf of the Burr XII distribution for $k, c, x>0$ is $G(x)=1-\left[1+x^{c}\right]^{-k}$. Then we define the Weibull Burr XII logarithmic 
(WBXIIL) cdf by

$$
F_{W B X I I L}(x)=\frac{\ln \left[1-p\left(1-e^{-\alpha\left[\left(1+x^{c}\right)^{k}-1\right]^{\beta}}\right)\right]}{\ln (1-p)} .
$$

The corresponding pdf of the WBXIIL distribution is given by

$$
f_{W B X I L}(x)=\frac{p \alpha \beta c k x^{c-1}\left(1+x^{c}\right)^{k-1}\left[\left(1+x^{c}\right)^{k}-1\right]^{\beta-1} e^{-\alpha\left[\left(1+x^{c}\right)^{k}-1\right]^{\beta}}}{\left[p\left(1-e^{-\alpha\left[\left(1+x^{c}\right)^{k}-1\right]^{\beta}}\right)-1\right] \ln (1-p)},
$$

where $\alpha>0, \beta>0, c>0, k>0,0<p<1$ and $x>0$.

Plots of the WBXIIL density and hazard function for the selected parameter values are given in Figure (5a) and (5b), respectively. The density and hazard functions for WBXIIL reveals various shapes for the selected parameter values, as shown in these plots. The graph of the hazard function shows an increasing, decreasing and bathtub shapes for selected values of the parameters. The density function also reveals left skewed, right skewed and a bell shaped curves for selected values of the parameters.

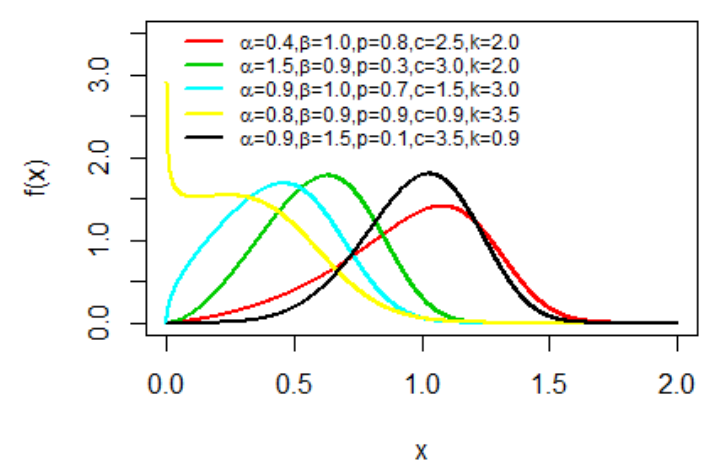

(a) Plot of WBXIIL density function

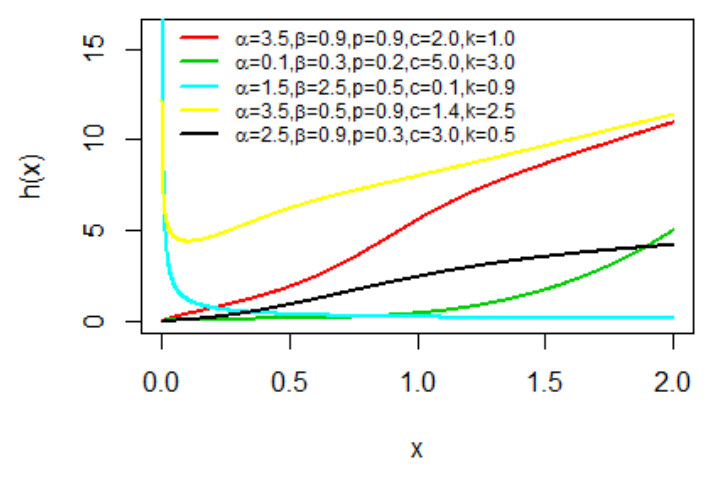

(b) Plot of WBXIIL hazard function

Figure 5. Plots of WBXIIL Density and Hazard Function

\subsection{Moments}

In this section, we present the moments of the WGL distribution. The first five non-central moments, standard deviation (SD), coefficients of variation (CV), skewness (CS), and kurtosis (CK) are also given. The $r^{\text {th }}$ moment of the WGL distribution is given by

$$
E\left(X^{r}\right)=\sum_{i, j=0}^{\infty} \omega_{i, j}(p) \int_{-\infty}^{\infty} x^{r} f_{W G}(x ; \alpha(j+1), \beta, \xi) d x=\sum_{i, j=0}^{\infty} \omega_{i, j}(p) E\left(Y^{r}\right),
$$

where $Y \sim W G(\alpha(j+1), \beta, \xi)$. To obtain the moment generating function (MGF) of the WGL distribution, recall the Taylor's series expansion of the function $e^{t x}=\sum_{j=0}^{\infty} \frac{(t x)^{j}}{j !}$, so that, we have

$$
M_{X}(t)=E\left(e^{t X}\right)=\sum_{n=0}^{\infty} \frac{t^{n}}{n !} E\left(X^{n}\right),
$$

where $E\left(X^{r}\right)$ is given by equation (30).

Table 3 lists the first five moments together with the standard deviation (SD or $\sigma$ ), coefficient of variation (CV), coefficient of skewness (CS) and coefficient of kurtosis (CK) of the WLLoL distribution for selected values of the parameters. These values can be determined numerically using R and MATLAB. The variance $\sigma^{2}$, standard deviation $\sigma, \mathrm{CV}, \mathrm{CS}$ and CK are given by

$$
\sigma^{2}=\mu_{2}^{\prime}-\mu^{2}, \quad C V=\frac{\sigma}{\mu}=\frac{\sqrt{\mu_{2}^{\prime}-\mu^{2}}}{\mu}=\sqrt{\frac{\mu_{2}^{\prime}}{\mu^{2}}-1},
$$




$$
C S=\frac{E\left[(X-\mu)^{3}\right]}{\left[E(X-\mu)^{2}\right]^{3 / 2}}=\frac{\mu_{3}^{\prime}-3 \mu \mu_{2}^{\prime}+2 \mu^{3}}{\left(\mu_{2}^{\prime}-\mu^{2}\right)^{3 / 2}}, \quad \text { and } \quad C K=\frac{E\left[(X-\mu)^{4}\right]}{\left[E(X-\mu)^{2}\right]^{2}}=\frac{\mu_{4}^{\prime}-4 \mu \mu_{3}^{\prime}+6 \mu^{2} \mu_{2}^{\prime}-3 \mu^{4}}{\left(\mu_{2}^{\prime}-\mu^{2}\right)^{2}},
$$

respectively.

Table 3. Table of Moments for Selected Parameters Values of the WLLoL Distribution

\begin{tabular}{c|ccccc}
\hline & $(1,3,2,0.4)$ & $(5,5,1,0.5)$ & $(1,1,2,0.5)$ & $(2,1,0.8,0.2)$ & $(0.2,3,2,0.3)$ \\
\hline$E(X)$ & 0.5506203 & 0.6949250 & 0.9782827 & 0.9321901 & 0.2473373 \\
$E\left(X^{2}\right)$ & 0.3783120 & 0.5056466 & 1.1865691 & 1.2085883 & 0.5895358 \\
$E\left(X^{3}\right)$ & 0.3017945 & 0.3816975 & 1.6631444 & 1.9394203 & 4.4465957 \\
$E\left(X^{4}\right)$ & 0.2696133 & 0.2970662 & 2.6013023 & 3.6456258 & 3.8262230 \\
$E\left(X^{5}\right)$ & 0.2639849 & 0.2373138 & 4.4472317 & 7.7742305 & 2232.8145375 \\
$S D$ & 0.2740972 & 0.1507508 & 0.4790951 & 0.5827606 & 0.7268838 \\
$C V$ & 0.4977971 & 0.2169311 & 0.4897307 & 0.6251521 & 2.9388358 \\
$C S$ & 0.5221681 & -0.3719849 & 0.4842317 & 0.9076005 & 10.5177681 \\
$C K$ & 3.0726259 & 2.9965418 & 3.0176472 & 3.9020649 & 249.4307164 \\
\hline
\end{tabular}

\subsection{Conditional Moments}

Conditional moments are very useful in probability and statistics, particularly in reliability and lifetime studies. The mean residual life function and vitality function can be readily obtained from the conditional moments. The $r^{\text {th }}$ conditional moments for the WGL distribution is given by

$$
\begin{aligned}
E\left(X^{r} \mid X>t\right) & =\frac{1}{\bar{F}_{W G L}(t)} \int_{t}^{\infty} x^{r} f_{W G L}(x ; \alpha(j+1), \beta, p, \xi) d x \\
& =\frac{1}{\bar{F}_{W G L}(t)} \sum_{j=0}^{\infty} \sum_{i=0}^{\infty} \omega_{i, j}(p) \int_{t}^{\infty} x^{r} \alpha(j+1) \beta g(x ; \xi)\left[\frac{G(x ; \xi)^{\beta-1}}{\bar{G}(x ; \xi)^{\beta+1}}\right] e^{-\alpha(j+1)\left[\frac{G(x ; \xi)}{G(x ; r)}\right]^{\beta}} d x \\
& =\frac{1}{\bar{F}_{W G L}(t)} \sum_{i=0}^{\infty} \sum_{j=0}^{\infty} \sum_{k=0}^{\infty} \sum_{m=0}^{\infty} \omega_{i, j}(p) \frac{(-1)^{k} \Gamma(\beta(k+1)+m+1)}{k ! m ! \Gamma(\beta(k+1)+1)} \\
& \times \alpha^{k+1} \beta(j+1) \int_{t}^{\infty} x^{r} g(x ; \xi) G(x ; \xi)^{\beta(k+1)+m-1} d x .
\end{aligned}
$$

\subsection{Order Statistics and Rényi Entropy}

This section contain the distribution of the order statistics and Rényi entropy for the WGL distribution.

\subsubsection{Order Statistics}

The pdf of the $i^{\text {th }}$ order statistic from the WGL pdf $f_{W G L}(x)$ is given by

$$
\begin{aligned}
g_{i: n}(x) & =\frac{n ! f_{W G L}(x)}{(i-1) !(n-i) !}\left[F_{W G L}(x)\right]^{i-1}\left[1-F_{W G L}(x)\right]^{n-i} \\
& =\frac{n ! f_{W G L}(x)}{(i-1) !(n-i) !} \sum_{j=0}^{n-i}(-1)^{j}\left(\begin{array}{c}
n-i \\
j
\end{array}\right)\left[F_{W G L}(x)\right]^{j+i-1}
\end{aligned}
$$

by using the binomial expansion $\left[1-F_{W G L}(x)\right]^{n-i}=\sum_{m=0}^{n-i}\left(\begin{array}{c}n-i \\ m\end{array}\right)(-1)^{m}\left[F_{W G L}(x)\right]^{m}$, so that

$$
\begin{aligned}
g_{i: n}(x) & =\frac{1}{B(i, n-i+1)} \sum_{m=0}^{n-i}\left(\begin{array}{c}
n-i \\
m
\end{array}\right) \frac{(-1)^{m}}{m+i}(m+i)\left[F_{W G L}(x)\right]^{m+i-1} f_{W G L}(x) \\
& =\sum_{m=0}^{n-i} w_{i, m} f_{m+i}(x),
\end{aligned}
$$

where $f_{m+i}(x)$ is the pdf of the exponentiated WGL distribution with parameters $\alpha, \beta, p$ and $(m+i), B(.,$.$) is the beta$ 
function and the weights $w_{i, m}$ are given by

$$
w_{i, m}=\frac{1}{B(i, n-i+1)} \frac{(-1)^{m}}{m+i}\left(\begin{array}{c}
n-i \\
m
\end{array}\right)=(-1)^{m}\left(\begin{array}{c}
m+i-1 \\
m
\end{array}\right)\left(\begin{array}{c}
n \\
m+i
\end{array}\right) .
$$

\subsubsection{Rényi Entropy}

Rényi entropy of the WGL distribution is given by

$$
I_{R}(v)=\frac{1}{1-v} \log \left(\int_{0}^{\infty}\left[f_{W G L}(x ; \alpha, \beta, p, \xi)\right]^{v} d x\right), v \neq 1, v>0 .
$$

Note that $\left[f_{W G L}(x ; \alpha, \beta, p, \xi)\right]^{v}=f_{W G L}^{v}(x)$ can be written as

$$
\begin{aligned}
f_{w G L}^{v}(x) & =\left(\frac{p \alpha \beta g(x ; \xi)\left[\frac{G\left(x ; \xi \beta^{\beta-1}\right.}{\bar{G}\left(x ; \xi \xi^{\beta+1}\right.}\right] e^{-\alpha\left[\frac{G(x ; \xi)}{G(x ; \xi)}\right]^{\beta}}}{\left[p\left(1-e^{-\alpha\left[\frac{G(x ; \xi)}{G(x ; \xi)}\right]^{\beta}}\right)-1\right] \ln (1-p)}\right)^{v} \\
& =\sum_{i=0}^{\infty} \sum_{j=0}^{\infty} \sum_{k=0}^{\infty} \sum_{m=0}^{\infty} \frac{(-1)^{j+k+v} \Gamma(i+v) \Gamma(v(\beta+1)+\beta k+m)}{j ! k ! m ! \Gamma(v) \Gamma(i-j+1) \Gamma(v(\beta+1)+\beta k)} \frac{p^{i+v} \alpha^{k+v} \beta^{v}(j+v)^{k}}{(\ln (1-p))^{v}} g(x ; \xi)^{v} G(x ; \xi)^{v(\beta-1)+\beta k+m} .
\end{aligned}
$$

Consequently, Rényi entropy for the WGL distribution reduces to

$$
\begin{aligned}
I_{R}(v) & =\frac{1}{1-v} \log \left[\sum_{i=0}^{\infty} \sum_{j=0}^{\infty} \sum_{k=0}^{\infty} \sum_{m=0}^{\infty} \frac{(-1)^{j+k+v} \Gamma(i+v) \Gamma(v(\beta+1)+\beta k+m)}{j ! k ! m ! \Gamma(v) \Gamma(i-j+1) \Gamma(v(\beta+1)+\beta k)}\right. \\
& \left.\times \frac{p^{i+v} \alpha^{k+v} \beta^{v}(j+v)^{k}}{(\ln (1-p))^{v}} \int_{0}^{\infty} g(x ; \xi)^{v} G(x ; \xi)^{v(\beta-1)+\beta k+m} d x\right], v>0, v \neq 1 .
\end{aligned}
$$

\subsection{Maximum Likelihood Estimation}

Let $X \sim W G L(p, \alpha, \beta)$ and $\boldsymbol{\Delta}=(p, \alpha, \beta, \xi)^{T}$ be the parameter vector. The log-likelihood $\ell=\ell(\Delta)$ based on a random sample of size $n$ is given by

$$
\begin{aligned}
\ell & =n \ln (p \alpha \beta)-n \ln (\ln (1-p))+\sum_{i=1}^{n} \ln \left[g\left(x_{i} ; \xi\right)\right]-\alpha \sum_{i=1}^{n} H\left(x_{i} ; \xi\right)^{\beta}+\beta \sum_{i=1}^{n} \ln \left[H\left(x_{i} ; \xi\right)\right]-\sum_{i=1}^{n} \ln \left[G\left(x_{i} ; \xi\right)\right] \\
& -\sum_{i=1}^{n} \ln \left[\bar{G}\left(x_{i} ; \xi\right)\right]-\sum_{i=1}^{n} \ln \left[p\left(1-e^{-\alpha H\left(x_{i} ; \xi^{\beta}\right.}\right)-1\right]
\end{aligned}
$$

where $H\left(x_{i} ; \xi\right)=G\left(x_{i} ; \xi\right) / \bar{G}\left(x_{i}, \xi\right)$. Elements of the score vector $U=\left(\frac{\partial \ell}{\partial p}, \frac{\partial \ell}{\partial \alpha}, \frac{\partial \ell}{\partial \beta}, \frac{\partial \ell}{\partial \xi_{\xi}}\right)$ are given by:

$$
\begin{gathered}
\frac{\partial \ell}{\partial p}=\frac{n}{p}-\frac{n}{(1-p) \ln (1-p)}-\sum_{i=1}^{n} \frac{1-e^{-\alpha H\left(x_{i} ; \xi^{\beta}\right.}}{p\left(1-e^{-\alpha H\left(x_{i} ; \xi^{\beta}\right.}\right)-1}, \\
\frac{\partial \ell}{\partial \alpha}=\frac{n}{\alpha}+\sum_{i=1}^{n} H\left(x_{i} ; \xi\right)^{\beta}-\sum_{i=1}^{n} \frac{p H\left(x_{i} ; \xi\right)^{\beta} e^{-\alpha H\left(x_{i} ; \xi\right)^{\beta}}}{p\left(1-e^{-\alpha H\left(x_{i} ; \xi^{\beta}\right.}\right)-1}, \\
\frac{\partial \ell}{\partial \beta}=\frac{n}{\beta}-\alpha \sum_{i=1}^{n} H\left(x_{i} ; \xi\right)^{\beta} \ln \left[H\left(x_{i} ; \xi\right)\right]+\sum_{i=1}^{n} \ln \left[H\left(x_{i} ; \xi\right)\right]+\sum_{i=1}^{n} \frac{p H\left(x_{i} ; \xi\right)^{\beta} \ln \left[H\left(x_{i} ; \xi\right)\right] e^{-\alpha H\left(x_{i} ; \xi\right)^{\beta}}}{p\left(1-e^{-\alpha H\left(x_{i} ; \xi\right)^{\beta}}\right)-1},
\end{gathered}
$$

and

$$
\begin{aligned}
\frac{\partial \ell}{\partial \xi_{k}} & =\sum_{i=1}^{n} \frac{\partial g\left(x_{i} ; \xi\right) / \partial \xi_{k}}{g\left(x_{i} ; \xi\right)}-\alpha \beta \sum_{i=1}^{n} H\left(x_{i} ; \xi\right)^{\beta-1} \frac{\partial H\left(x_{i} ; \xi\right)}{\partial \xi_{k}}+\beta \sum_{i=1}^{n} \frac{\partial H\left(x_{i} ; \xi\right) / \partial \xi_{k}}{H\left(x_{i} ; \xi\right)}-\sum_{i=1}^{n} \frac{\partial G\left(x_{i} ; \xi\right) / \partial \xi_{k}}{G\left(x_{i} ; \xi\right)} \\
& -\sum_{i=1}^{n} \frac{\partial \bar{G}\left(x_{i} ; \xi\right) / \partial \xi_{k}}{\bar{G}\left(x_{i} ; \xi\right)}-\sum_{i=1}^{n} \frac{p \alpha \beta H\left(x_{i} ; \xi\right)^{\beta-1} e^{-\alpha H\left(x_{i} ; \xi^{\beta}\right.} \partial H\left(x_{i} ; \xi\right) / \partial \xi_{k}}{p\left(1-e^{-\alpha H\left(x_{i} ; \xi^{\beta}\right.}\right)-1}
\end{aligned}
$$


respectively. The equations obtained by setting the above partial derivatives to zero are not in closed form and the values of the parameters $p, \alpha, \beta, \xi_{k}$ must be found via iterative methods. The maximum likelihood estimates of the parameters, denoted by $\hat{\boldsymbol{\Delta}}$ is obtained by solving the non-linear equation $\left(\frac{\partial \ell}{\partial p}, \frac{\partial \ell}{\partial \alpha}, \frac{\partial \ell}{\partial \beta}, \frac{\partial \ell}{\partial \xi_{k}}\right)^{T}=\mathbf{0}$, using a numerical method such as Newton-Raphson procedure. The Fisher information matrix is given by $\mathbf{I}(\boldsymbol{\Delta})=\left[\mathbf{I}_{\theta_{i}, \theta_{j}}\right]_{t X t}=E\left(-\frac{\partial^{2} \ell}{\partial \theta_{i} \partial \theta_{j}}\right), i, j=1,2, \ldots, t$, can be numerically obtained by NLMIXED in SAS or R software. The total Fisher information matrix $n \mathbf{I}(\Delta)$ can be approximated by

$$
\mathbf{J}_{n}(\hat{\boldsymbol{\Lambda}}) \approx\left[-\left.\frac{\partial^{2} \ell}{\partial \theta_{i} \partial \theta_{j}}\right|_{\Delta=\hat{\mathbf{\Delta}}}\right]_{t X t}, \quad i, j=1,2, \ldots, t,
$$

where $t$ is the number of model parameters. For a given set of observations, the matrix given in equation (33) is obtained after the convergence of the Newton-Raphson procedure via NLMIXED in SAS or R software.

\section{Simulation Study}

In this section, we examine the performance of the WLLoL distribution by conducting various simulations for different sample sizes $(n=25,50,100,200,400,800)$ via the R package. We simulate 1000 samples for the true parameters values given in Table 4. Table 4 lists the mean MLEs of the four model parameters along with the respective biases and root mean squared errors (RMSEs). The bias and RMSE are given by:

$$
\operatorname{Bias}(\hat{\theta})=\frac{\sum_{i=1}^{N} \hat{\theta}_{i}}{N}-\theta, \quad \text { and } \quad \operatorname{RMSE}(\hat{\theta})=\sqrt{\frac{\sum_{i=1}^{N}\left(\hat{\theta}_{i}-\theta\right)^{2}}{N}},
$$

respectively, where $\theta$ stand for the parameters $c, \alpha, \beta$ and $p$.

\begin{tabular}{|c|c|c|c|c|c|c|c|c|c|c|}
\hline \multirow[b]{2}{*}{ Parameter } & \multirow[b]{2}{*}{ Sample Size } & \multicolumn{3}{|c|}{$(0.4,2.0,2.0,0.1)$} & \multicolumn{3}{|c|}{$(2.0,0.1,2.0,0.1)$} & \multicolumn{3}{|c|}{$(2.0,2.0,2.0,0.4)$} \\
\hline & & Mean & RMSE & Bias & Mean & RMSE & Bias & Mean & RMSE & Bias \\
\hline \multirow[t]{6}{*}{$c$} & 25 & 0.4034 & 0.0782 & 0.0034 & 1.9342 & 0.1763 & -0.0658 & 1.9686 & 0.1944 & -0.0314 \\
\hline & 50 & 0.3824 & 0.0758 & -0.0176 & 1.9131 & 0.1502 & -0.0869 & 1.9298 & 0.1463 & -0.0702 \\
\hline & 100 & 0.3795 & 0.0596 & -0.0205 & 1.9308 & 0.1191 & -0.0692 & 1.9472 & 0.1176 & -0.0528 \\
\hline & 200 & 0.3827 & 0.0401 & -0.0173 & 1.9349 & 0.1021 & -0.0651 & 1.9521 & 0.0966 & -0.0479 \\
\hline & 400 & 0.3836 & 0.0360 & -0.0164 & 1.9443 & 0.0837 & -0.0557 & 1.9653 & 0.0770 & -0.0347 \\
\hline & 800 & 0.3884 & 0.0230 & -0.0116 & 1.9602 & 0.0606 & -0.0398 & 1.9777 & 0.0569 & -0.0223 \\
\hline \multirow[t]{6}{*}{$\alpha$} & 25 & 2.6665 & 1.0093 & 0.6665 & 0.1992 & 0.1520 & 0.0992 & 2.5594 & 0.8876 & 0.5594 \\
\hline & 50 & 2.5982 & 0.8251 & 0.5982 & 0.1859 & 0.1382 & 0.08589 & 2.4855 & 0.7561 & 0.4855 \\
\hline & 100 & 2.5633 & 0.7682 & 0.5633 & 0.1656 & 0.0996 & 0.0656 & 2.3924 & 0.6420 & 0.3924 \\
\hline & 200 & 2.4465 & 0.5989 & 0.4465 & 0.1547 & 0.0807 & 0.0547 & 2.3197 & 0.5205 & 0.3197 \\
\hline & 400 & 2.2975 & 0.4063 & 0.2975 & 0.1406 & 0.0595 & 0.0406 & 2.2049 & 0.3915 & 0.2049 \\
\hline & 800 & 2.2037 & 0.2822 & 0.2037 & 0.1265 & 0.0386 & 0.0265 & 2.1297 & 0.2788 & 0.1297 \\
\hline \multirow[t]{6}{*}{$\beta$} & 25 & 1.8879 & 0.3156 & -0.1121 & 1.9342 & 0.1763 & -0.0658 & 1.9686 & 0.1944 & -0.0314 \\
\hline & 50 & 1.9554 & 0.2934 & -0.0446 & 1.9131 & 0.1502 & -0.0869 & 1.9298 & 0.1463 & -0.0702 \\
\hline & 100 & 1.9571 & 0.2334 & -0.0429 & 1.9308 & 0.1191 & -0.0692 & 1.9472 & 0.1176 & -0.0528 \\
\hline & 200 & 1.9544 & 0.1536 & -0.0456 & 1.9349 & 0.1021 & -0.0651 & 1.9521 & 0.09656 & -0.0479 \\
\hline & 400 & 1.9922 & 0.1291 & -0.0078 & 1.9443 & 0.0837 & -0.0557 & 1.9653 & 0.0770 & -0.0347 \\
\hline & 800 & 1.9870 & 0.0633 & -0.0130 & 1.9602 & 0.0606 & -0.0398 & 1.9777 & 0.0569 & -0.0223 \\
\hline \multirow[t]{6}{*}{$p$} & 25 & 0.6461 & 0.6045 & 0.5461 & 0.6271 & 0.6004 & 0.5271 & 0.6646 & 0.3683 & 0.2646 \\
\hline & 50 & 0.6310 & 0.5866 & 0.5310 & 0.5870 & 0.5562 & 0.4870 & 0.6508 & 0.3534 & 0.2508 \\
\hline & 100 & 0.6038 & 0.5682 & 0.5038 & 0.5639 & 0.5333 & 0.4639 & 0.6040 & 0.3313 & 0.2040 \\
\hline & 200 & 0.5421 & 0.5092 & 0.4421 & 0.5139 & 0.4948 & 0.4139 & 0.5833 & 0.3084 & 0.1833 \\
\hline & 400 & 0.4546 & 0.4222 & 0.3546 & 0.4542 & 0.4300 & 0.3542 & 0.5288 & 0.2691 & 0.1288 \\
\hline & 800 & 0.3710 & 0.3424 & 0.2710 & 0.3739 & 0.3463 & 0.2739 & 0.4832 & 0.2334 & 0.0832 \\
\hline
\end{tabular}

Table 4. Monte Carlo Simulation Results

From the tabulated results, we note that as the sample size $n$ increases, the mean estimates of the parameters tend to be closer to the true parameter values, since RMSEs decay toward zero. 


\section{Applications}

In this section, we present examples to illustrate the flexibility of the WGL distribution by fitting some special cases including the WLLoL, WWL and WBXIIL distributions and their sub-models for data modelling. The maximum likelihood estimates (MLEs) of the WLLoL, WWL and WBXIIL parameters are computed by maximizing the objective function via the subroutine NLMIXED in SAS as well as the function nlm in R. The estimated values of the parameters (standard error in parenthesis), -2log-likelihood statistic, Akaike Information Criterion, $A I C=2 p-2 \ln (L)$, and Bayesian Information Criterion, $B I C=p \ln (n)-2 \ln (L)$, and Consistent Akaike Information Criterion, AICC $=A I C+2 \frac{p(p+1)}{n-p-1}$, where $L=L(\hat{\Delta})$ is the value of the likelihood function evaluated at the parameter estimates, $n$ is the number of observations, and $p$ is the number of estimated parameters are presented. Tables 5, 6 and 7 shows the results for the data set for WLLoL, WWL, WBXIIL and their sub-models. The goodness-of-fit statistics: Cramer von Mises $\left(W^{*}\right)$ and Andersen-Darling $\left(A^{*}\right)$, described by Chen \& Balakrishnan (1985), as well as Kolmogorov Simirnov (KS) and its p-value, and sum of squares (SS) from the probability plots are also presented in the tables. These statistics can be used to verify which distribution fits better to the data. In general, the smaller the values of $W^{*}, A^{*}$ and KS goodness-of-fit statistics, the better the fit.

We also compare the fit of the WLLoL distribution to the non-nested models: Gamma-Dagum (GDa) (see Rodrigues and Silva (2015)), and the new modified Weibull (NMW) distribution introduced by Doostmoradi, Zadkarami \& Roshani (2014). The pdf of the four parameter GDa, and NMW distributions are given in equation (34) and equation (35), respectively, that is,

$$
g_{G D a}(x)=\frac{\lambda \delta \beta^{\alpha}}{\Gamma(\alpha)} x^{-\delta-1}\left(1+\lambda x^{-\delta}\right)^{-\beta-1}\left[\log \left(1+\lambda x^{-\delta}\right)\right]^{\alpha-1}, \quad x>0,
$$

and

$$
g_{N M W}(x)=\left(\alpha \gamma x^{\gamma-1} e^{\alpha x^{\gamma}}+\lambda \beta x^{\lambda-1} e^{-\beta x^{\lambda}}\right) e^{-e^{\alpha x^{\gamma}}+e^{-\beta x^{\lambda}}}, \quad x>0 .
$$

The fits of the WWL and WBXIIL distributions were also compared to the non-nested models: Gamma-Dagum (GD) (Oluyede, Huang \& Pararai (2015)) and beta exponentiated Weibull (BEW) distribution (Cordeiro et al. (2013)) for the fitted data set. The pdf of the GD and BEW distribution are given in equation (36) and equation (37), respectively, that is,

$$
\begin{aligned}
g_{G D}(x) & =\frac{\lambda \beta \delta x^{-\delta-1}}{\theta^{\alpha} \Gamma(\alpha)}\left(1+\lambda x^{-\delta}\right)^{-\beta-1}\left(-\log \left(1-\left(1+\lambda x^{-\delta}\right)^{-\beta}\right)\right)^{\alpha-1} \\
& \times\left[1-\left(1+\lambda x^{-\delta}\right)^{-\beta}\right]^{1 / \theta-1}, \quad x>0,
\end{aligned}
$$

and

$$
g_{B E W}(x)=\frac{\alpha \beta \lambda^{\beta}}{B(a, b)} x^{\beta-1} e^{-(\lambda x)^{\beta}}\left(1-e^{-(\lambda x)^{\beta}}\right)^{\alpha a-1}\left(1-\left(1-e^{-(\lambda x)^{\beta}}\right)^{\alpha}\right)^{b-1}, \quad x>0 .
$$

We can use the likelihood ratio (LR) test to compare the fit of the WLLoL, WWL and WBXIIL distribution with their sub-models for a given data set. For example, to test $\beta=1$, the LR statistic is $\omega=2[\ln (L(\hat{c}, \hat{\alpha}, \hat{\beta}, \hat{p}))-\ln (L(\tilde{\alpha}, 1, \tilde{c}, \tilde{p}))]$, where $\hat{\alpha}, \hat{\beta}, \hat{c}$, and $\hat{p}$ are the unrestricted estimates, and $\tilde{\alpha}, \tilde{c}$, and $\tilde{p}$ are the restricted estimates. The LR test rejects the null hypothesis if $\omega>\chi_{\epsilon}^{2}$, where $\chi_{\epsilon}^{2}$ denote the upper $100 \epsilon \%$ point of the $\chi^{2}$ distribution with 1 degrees of freedom.

The plots of the fitted densities, the histogram of the data and probability plots (Chambers et al. (1983)) are given in Figure 6 , and Figure 7 for WLLoL, WWL and WBXIIL distributions. For the probability plot, we plotted $G_{W G L}\left(x_{(j)} ; \hat{\alpha}, \hat{\beta}, \hat{c}, \hat{p}\right)$ against $\frac{j-0.375}{n+0.25}, j=1,2, \cdots, n$, where $x_{(j)}$ are the ordered values of the observed data. The measure of closeness is given by the sum of squares $S S=\sum_{j=1}^{n}\left[G_{W G L}\left(x_{(j)}\right)-\left(\frac{j-0.375}{n+0.25}\right)\right]^{2}$.

\subsection{The Fracture Toughness of Alumina Data}

This data consist of 119 observations of the fracture toughness of Alumina $\left(\mathrm{Al}_{2} \mathrm{O}_{3}\right)$ (in the units of $\mathrm{MPa} \mathrm{m}^{1 / 2}$ ), from Nadarajah \& Kotz (2008). These data are 5.50, 5.00, 4.90, 6.40, 5.10, 5.20, 5.20, 5.00, 4.70, 4.00, 4.50, 4.20, 4.10, 4.56, $5.01,4.70,3.13,3.12,2.68,2.77,2.70,2.36,4.38,5.73,4.35,6.81,1.91,2.66,2.61,1.68,2.04,2.08,2.13,3.80,3.73$, $3.71,3.28,3.90,4.00,3.80,4.10,3.90,4.05,4.00,3.95,4.00,4.50,4.50,4.20,4.55,4.65,4.10,4.25,4.30,4.50,4.70$, $5.15,4.30,4.50,4.90,5.00,5.35,5.15,5.25,5.80,5.85,5.90,5.75,6.25,6.05,5.90,3.60,4.10,4.50,5.30,4.85,5.30$, $5.45,5.10,5.30,5.20,5.30,5.25,4.75,4.50,4.20,4.00,4.15,4.25,4.30,3.75,3.95,3.51,4.13,5.40,5.00,2.10,4.60$, 3.20, 2.50, 4.10, 3.50, 3.20, 3.30, 4.60, 4.30, 4.30, 4.50, 5.50, 4.60, 4.90, 4.30, 3.00, 3.40, 3.70, 4.40, 4.90, 4.90, 5.00.

The LR test statistic of the hypothesis $H_{0}$ : WLLo against $H_{a}$ : WLLoL and $H_{0}$ :LLo against $H_{a}$ : WLLoL are 9.87 (p-value $=0.0017)$ and 447.82 (p-value $<0.0001)$. We can conclude that there are significant difference between the fit of the WLLoL and WLLo distributions as well between WLLoL and LLo distributions. The WLLoL distribution is also better 
than the non-nested NMW and GDa distributions based on all the statistics presented in Table 5. The probability plots of the compared distributions based on the fracture toughness of alumina data set is shown in Figure 6. The value of SS from the probability plots is smallest for the WLLoL distribution.

Table 5. Parameter estimates and goodness-of-fit statistics for various fitted models for the alumina data set

\begin{tabular}{|c|c|c|c|c|c|c|c|c|c|c|c|c|c|c|}
\hline \multirow[b]{2}{*}{ Model } & \multicolumn{4}{|c|}{ Estimates } & \multicolumn{10}{|c|}{ Statistics } \\
\hline & $\alpha$ & $\beta$ & $c$ & $p$ & $-2 \log L$ & $A I C$ & $A I C C$ & CAIC & $B I C$ & $W^{*}$ & $A^{*}$ & $K S$ & $P-$ value & $S S$ \\
\hline WLLoL & $\begin{array}{c}0.0069 \\
(0.0024)\end{array}$ & $\begin{array}{c}1.3128 \\
(0.4569)\end{array}$ & $\begin{array}{c}2.8298 \\
(0.9848)\end{array}$ & $\begin{array}{c}0.9504 \\
(0.0386)\end{array}$ & 337.74 & 345.74 & 346.09 & 346.09 & 356.85 & 0.0806 & 0.4635 & 0.0776 & 0.4700 & 0.1002 \\
\hline WLLo & $\begin{array}{c}0.0026 \\
(0.0003)\end{array}$ & $\begin{array}{c}0.8830 \\
(0.1418)\end{array}$ & $\begin{array}{c}4.4099 \\
(0.7081)\end{array}$ & - & 347.61 & 353.61 & 353.82 & 353.82 & 361.95 & 0.1246 & 0.7719 & 0.1583 & 0.0051 & 0.6421 \\
\hline LLo & $\begin{array}{l}- \\
-\end{array}$ & $\begin{array}{l}- \\
-\end{array}$ & $\begin{array}{c}1.0683 \\
(0.0758)\end{array}$ & - & 785.56 & 787.56 & 787.59 & 787.59 & 790.34 & 0.6487 & 3.8299 & 0.6687 & 0.0000 & 19.3713 \\
\hline$N M W$ & $\begin{array}{c}\alpha \\
0.0032 \\
(0.0015)\end{array}$ & $\begin{array}{c}\beta \\
3.7000 \\
(0.2485)\end{array}$ & $\begin{array}{c}\gamma \\
3.4182 \\
(0.2843)\end{array}$ & $\begin{array}{c}\lambda \\
4.1000 \\
(0.2664)\end{array}$ & 581.10 & 589.10 & 589.45 & 589.45 & 600.21 & 0.6448 & 3.9230 & 0.6391 & 0.0000 & 16.1455 \\
\hline$G D a$ & $\begin{array}{c}\alpha \\
12.3287 \\
(9.8449)\end{array}$ & $\begin{array}{c}\beta \\
7.0959 \\
(4.1078)\end{array}$ & $\begin{array}{c}\lambda \\
131.1517 \\
(97.3091)\end{array}$ & $\begin{array}{c}\delta \\
2.3494 \\
(0.6314)\end{array}$ & 354.65 & 362.65 & 363.00 & 363.00 & 373.77 & 0.3832 & 2.3266 & 0.1273 & 0.0423 & 0.38462 \\
\hline
\end{tabular}

Table 6. Parameter estimates and goodness-of-fit statistics for various fitted models for the alumina data set

\begin{tabular}{|c|c|c|c|c|c|c|c|c|c|c|c|c|c|c|c|}
\hline \multirow[b]{2}{*}{ Model } & \multicolumn{5}{|c|}{ Estimates } & \multicolumn{10}{|c|}{ Statistics } \\
\hline & $\alpha$ & $\beta$ & $\lambda$ & $\gamma$ & $p$ & $-2 \log L$ & $A I C$ & $A I C C$ & CAIC & $B I C$ & $W^{*}$ & $A^{*}$ & $K S$ & $P$-value & $S S$ \\
\hline WWL & $\begin{array}{c}0.0113 \\
(0.0080)\end{array}$ & $\begin{array}{c}2.4326 \\
(0.9862)\end{array}$ & $\begin{array}{c}0.5639 \\
(0.2235)\end{array}$ & $\begin{array}{c}0.8259 \\
(0.2283)\end{array}$ & $\begin{array}{c}0.4205 \\
(0.7698)\end{array}$ & 339.05 & 349.05 & 349.41 & 349.59 & 362.95 & 0.0806 & 0.4778 & 0.0691 & 0.6204 & 0.0939 \\
\hline$W W$ & $\begin{array}{c}0.0081 \\
(0.0038)\end{array}$ & $\begin{array}{c}0.5266 \\
(0.2169)\end{array}$ & $\begin{array}{c}1.7466 \\
(0.7487)\end{array}$ & $\begin{array}{c}1.0542 \\
(0.1195)\end{array}$ & $\begin{array}{l}- \\
-\end{array}$ & 345.49 & 353.49 & 353.84 & 353.84 & 364.61 & 0.1155 & 0.7030 & 0.0686 & 0.6295 & 0.1408 \\
\hline$W$ & - & $\begin{array}{l}- \\
-\end{array}$ & $\begin{array}{c}0.0023 \\
(0.0002)\end{array}$ & $\begin{array}{c}3.9908 \\
(0.0800)\end{array}$ & $\begin{array}{l}- \\
-\end{array}$ & 345.85 & 349.85 & 349.95 & 349.95 & 355.41 & 0.1200 & 0.7433 & 0.1541 & 0.0070 & 0.6008 \\
\hline$B E W$ & $\begin{array}{c}\alpha \\
1.2888 \\
(0.1667)\end{array}$ & $\begin{array}{c}\beta \\
4.0334 \\
(0.0021)\end{array}$ & $\begin{array}{c}a \\
1.0906 \\
(0.2270)\end{array}$ & $\begin{array}{c}b \\
0.1413 \\
(0.0143)\end{array}$ & $\begin{array}{c}\lambda \\
0.3579 \\
(0.0018)\end{array}$ & 341.69 & 351.69 & 352.22 & 352.22 & 365.59 & 0.1598 & 0.9804 & 0.1359 & 0.0248 & 0.4328 \\
\hline$G D$ & $\begin{array}{c}\alpha \\
0.2374 \\
(0.0261)\end{array}$ & $\begin{array}{c}\beta \\
17.5836 \\
(0.1046)\end{array}$ & $\begin{array}{c}\theta \\
0.0054 \\
(0.0025)\end{array}$ & $\begin{array}{c}\delta \\
2.0618 \\
(0.0712)\end{array}$ & $\begin{array}{c}\lambda \\
12.8960 \\
(0.0771)\end{array}$ & 339.56 & 349.56 & 349.92 & 350.09 & 363.46 & 0.3169 & 1.9015 & 0.8138 & 0.0000 & 31.6024 \\
\hline
\end{tabular}

Table 7. Parameter estimates and goodness-of-fit statistics for various fitted models for the alumina data set

\begin{tabular}{|c|c|c|c|c|c|c|c|c|c|c|c|c|c|c|c|}
\hline \multirow[b]{2}{*}{ Model } & \multicolumn{5}{|c|}{ Estimates } & \multicolumn{10}{|c|}{ Statistics } \\
\hline & $\alpha$ & $\beta$ & $c$ & $k$ & $p$ & $-2 \log L$ & $A I C$ & $A I C C$ & $C A I C$ & $B I C$ & $W^{*}$ & $A^{*}$ & $K S$ & $P$-value & $S S$ \\
\hline WBXIIL & $\begin{array}{c}0.0098 \\
(0.0051)\end{array}$ & $\begin{array}{c}2.0215 \\
(0.7461)\end{array}$ & $\begin{array}{c}2.4862 \\
(0.9254)\end{array}$ & $\begin{array}{c}0.7053 \\
(0.2630)\end{array}$ & $\begin{array}{c}0.9451 \\
(0.0551)\end{array}$ & 337.15 & 347.15 & 347.68 & 347.68 & 361.05 & 0.0793 & 0.4579 & 0.0797 & 0.4367 & 0.1020 \\
\hline WBXII & $\begin{array}{c}0.0026 \\
(0.0003)\end{array}$ & $\begin{array}{c}1.1417 \\
(0.2226)\end{array}$ & $\begin{array}{c}3.4637 \\
(0.5920)\end{array}$ & $\begin{array}{c}0.9857 \\
(0.1835)\end{array}$ & $\begin{array}{l}- \\
-\end{array}$ & 347.48 & 355.48 & 355.83 & 355.83 & 366.59 & 0.1248 & 0.7730 & 0.1573 & 0.0055 & 0.6309 \\
\hline$B X I I$ & $\begin{array}{l}- \\
- \\
\end{array}$ & - & $\begin{array}{c}6.6399 \\
(3.8950) \\
\end{array}$ & $\begin{array}{c}0.1052 \\
(0.0624) \\
\end{array}$ & - & 664.47 & 668.47 & 668.57 & 668.57 & 674.02 & 0.9703 & 5.5702 & 0.4306 & 0.0000 & 7.7877 \\
\hline$B E W$ & $\begin{array}{c}\alpha \\
1.2888 \\
(0.1667)\end{array}$ & $\begin{array}{c}\beta \\
4.0334 \\
(0.0021)\end{array}$ & $\begin{array}{c}a \\
1.0906 \\
(0.2270)\end{array}$ & $\begin{array}{c}b \\
0.1413 \\
(0.0143)\end{array}$ & $\begin{array}{c}\lambda \\
0.3579 \\
(0.0018)\end{array}$ & 341.69 & 351.69 & 352.22 & 352.22 & 365.59 & 0.1598 & 0.9804 & 0.1359 & 0.0248 & 0.4328 \\
\hline$G D$ & $\begin{array}{c}\alpha \\
0.2374 \\
(0.0261)\end{array}$ & $\begin{array}{c}\beta \\
17.5836 \\
(0.1046)\end{array}$ & $\begin{array}{c}\theta \\
0.0054 \\
(0.0025)\end{array}$ & $\begin{array}{c}\delta \\
2.0618 \\
(0.0712)\end{array}$ & $\begin{array}{c}\lambda \\
12.8960 \\
(0.0771)\end{array}$ & 339.56 & 349.56 & 349.92 & 350.09 & 363.46 & 0.3169 & 1.9015 & 0.8138 & 0.0000 & 31.6024 \\
\hline
\end{tabular}

The LR test statistic of the hypothesis $H_{0}$ : WW against $H_{a}$ : WWL and $H_{0}: \mathrm{W}$ against $H_{a}$ : WWL are 6.44 (p-value $=0.011)$ and 6.8 (p-value $=0.079)$. We can conclude that there is significant difference between the fit of the WWL and WW distributions. However, there is a significance difference between the fits of the WWL and W distributions at the 10\% significance level. The values of the goodness-of-fit statistics $W^{*}, A^{*}$ and KS in Table 6 point to the WWL distribution as the better fit. The probability plots of the compared distributions based on the fracture toughness of alumina data set is shown in Figure 7. The value of SS from the probability plots in Table 6 is smallest for the WWL distribution.

The LR test statistic of the hypothesis $H_{0}$ : WBXII against $H_{a}$ : WBXIIL and $H_{0}$ :BXII against $H_{a}$ : WBXIIL are 8.33 (p-value $=0.0039)$ and $321.32(\mathrm{p}$-value $<0.0001)$. We can conclude that there is significant difference between the fit of WBXIIL and WBXII distributions and also between the fits of WBXIIL and BXII distributions. The WWL and WBXIIL distributions also seemed to be better than the non-nested BEW and GD distributions based on the values of the statistics in Table 6 and Table 7, respectively. Also, the probability plots of the fitted models based on the fracture toughness of 
alumina data set is shown in Figure 7. The value of SS from the probability plots in Table 7 is smallest for the WBXIIL distribution.

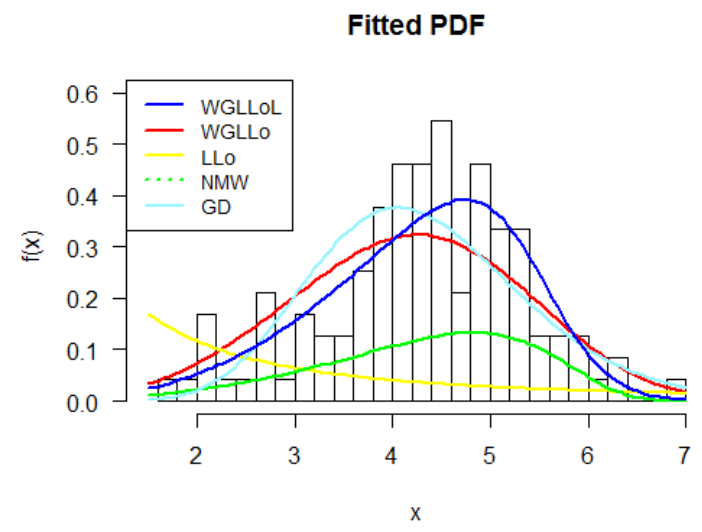

\section{The Graph of Observed vs Expected Probability}

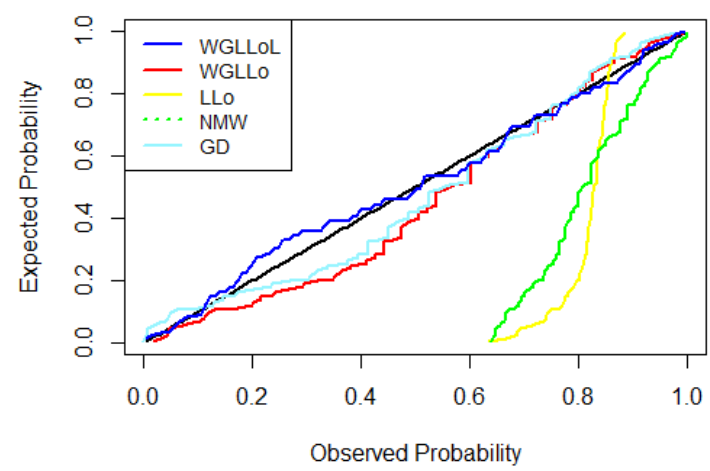

Figure 6. Fitted densities and probability plots fracture toughness of Alumina data

Fitted PDF

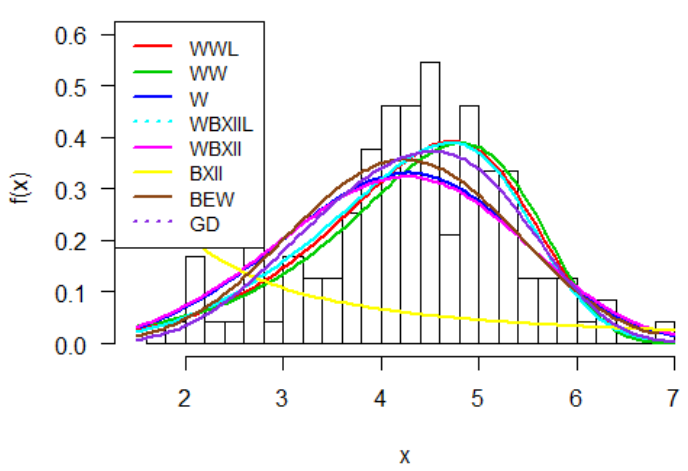

The Graph of Observed vs Expected Probability

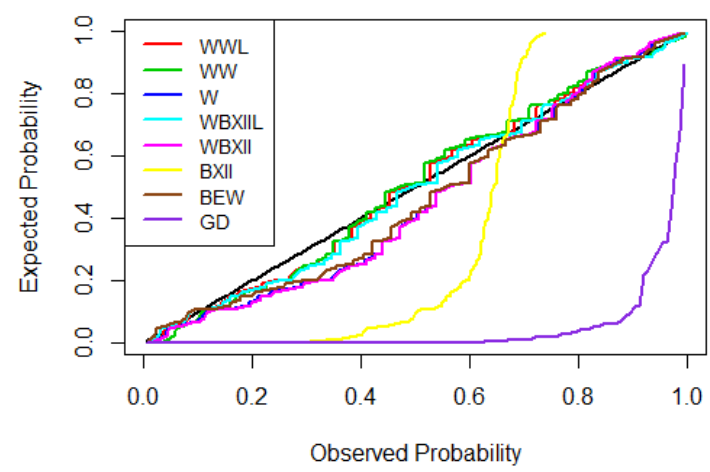

Figure 7. Fitted densities and probability plots for fracture toughness of Alumina data

\subsection{Fatigue Time of 101 6061-T6 Aluminum Coupons Data}

The second data consist of 101 observations. The data was was presented in Birnbaum \& Saunders (1969) and correspond to the fatigue time of $1016061-\mathrm{T} 6$ aluminum coupons cut parallel to the direction of rolling and oscillated at 18 cycles per second (cps). The data are: 70, 90, 96, 97, 99, 100, 103, 104, 104, 105, 107, 108, 108, 108, 109, 109, 112, 112, 113, $114,114,114,116,119,120,120,120,121,121,123,124,124,124,124,124,128,128,129,129,130,130,130,131$, $131,131,131,131,132,132,132,133,134,134,134,134,134,136,136,137,138,138,138,139,139,141,141,142$, $142,142,142,142,142,144,144,145,146,148,148,149,151,151,152,155,156,157,157,157,157,158,159,162$, $163,163,164,166,166,168,170,174,196,212$.

For this data, we fit the WWL distribution and its sub-models and compare it with the non-nested BEW and GD distributions. The LR test statistic of the hypothesis $H_{0}$ : WBXII against $H_{a}$ : WBXIIL and $H_{0}$ :BXII against $H_{a}$ : WBXIIL are 17.61 (p-value $<0.0001$ ) and 81.38 (p-value $<0.0001$ ). We can conclude that there is significant difference between WBXIIL and WBXII distributions and also between WBXIIL and BXII distributions. The WWL distribution also seemed to be better than the non-nested BEW and GD distributions based on the values of the AIC, BIC, KS statistic and its pvalue in Table 8 . The probability plots of the fitted distributions based on the aluminium coupon data set is shown in Figure 8 . The value of SS for the probability plots in Table 8 is smallest for the WWL distribution. 
Table 8. Parameter estimates and goodness-of-fit statistics for various fitted models for the aluminum coupons data set

\begin{tabular}{|c|c|c|c|c|c|c|c|c|c|c|c|c|c|c|c|}
\hline \multirow[b]{2}{*}{ Model } & \multicolumn{5}{|c|}{ Estimates } & \multicolumn{10}{|c|}{ Statistics } \\
\hline & $\alpha$ & $\beta$ & $\lambda$ & $\gamma$ & $p$ & $-2 \log L$ & $A I C$ & $A I C C$ & CAIC & $B I C$ & $W^{*}$ & $A^{*}$ & $K S$ & $P-$ value & $S S$ \\
\hline WWL & $\begin{array}{c}0.0060 \\
(0.0037)\end{array}$ & $\begin{array}{c}3.1600 \\
(0.9867)\end{array}$ & $\begin{array}{c}0.0873 \\
(0.1541)\end{array}$ & $\begin{array}{c}0.6264 \\
(0.3203)\end{array}$ & $\begin{array}{c}0.8732 \\
(0.3322)\end{array}$ & 938.49 & 948.49 & 948.91 & 949.12 & 961.56 & 0.2685 & 1.6496 & 0.1256 & 0.0825 & 0.3830 \\
\hline$W W$ & $\begin{array}{c}0.0022 \\
(0.0002)\end{array}$ & $\begin{array}{c}0.9664 \\
(0.1862)\end{array}$ & $\begin{array}{c}0.1288 \\
(0.0242)\end{array}$ & $\begin{array}{c}0.7853 \\
(0.0426)\end{array}$ & - & 946.78 & 954.78 & 955.19 & 955.19 & 965.24 & 0.2152 & 1.3850 & 0.1645 & 0.0085 & 0.8065 \\
\hline$W$ & $\begin{array}{l}- \\
-\end{array}$ & - & $\begin{array}{c}0.0036 \\
(0.0008)\end{array}$ & $\begin{array}{c}1.1516 \\
(0.0467)\end{array}$ & $\begin{array}{l}- \\
-\end{array}$ & 1163.38 & 1167.38 & 1167.50 & 1167.50 & 1172.61 & 0.0604 & 0.3698 & 0.4785 & 0.0000 & 6.6708 \\
\hline$B E W$ & $\begin{array}{c}\alpha \\
0.2058 \\
(0.1171) \\
\end{array}$ & $\begin{array}{c}\beta \\
1.3288 \\
(0.0037)\end{array}$ & $\begin{array}{c}a \\
0.6260 \\
(0.1633)\end{array}$ & $\begin{array}{c}b \\
0.0504 \\
(0.0057)\end{array}$ & $\begin{array}{c}\lambda \\
0.0675 \\
(0.0027)\end{array}$ & 1160.27 & 1170.27 & 1170.69 & 1170.90 & 1183.35 & 0.0543 & 0.3424 & 0.4956 & 0.0000 & 7.1190 \\
\hline$G D$ & $\begin{array}{c}\alpha \\
3.8329 \\
(0.3355)\end{array}$ & $\begin{array}{c}\beta \\
7.1388 \\
(0.0219)\end{array}$ & $\begin{array}{c}\theta \\
6.8900 \\
(0.5780)\end{array}$ & $\begin{array}{c}\delta \\
7.0515 \\
(0.0031)\end{array}$ & $\begin{array}{c}\lambda \\
17.1780 \\
(0.0568)\end{array}$ & 1335.39 & 1345.39 & 1345.81 & 1346.02 & 1358.47 & 0.0992 & 0.5720 & 0.8056 & 0.0000 & 20.3925 \\
\hline
\end{tabular}

Fitted PDF

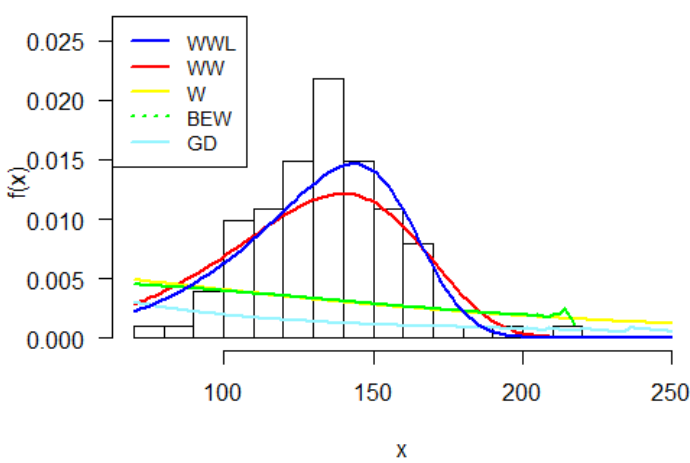

The Graph of Observed vs Expected Probability

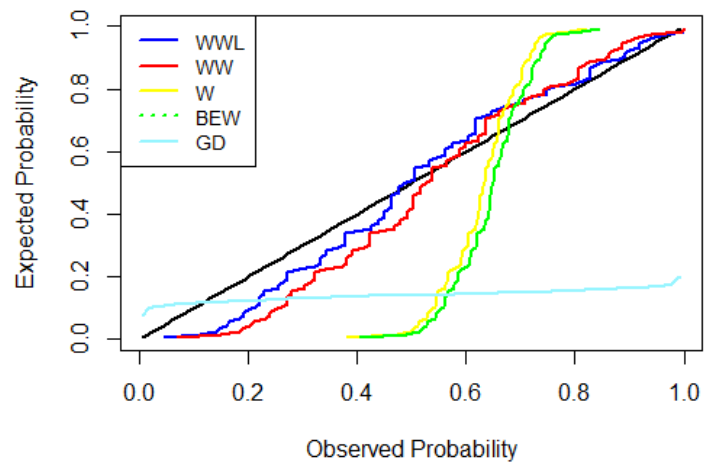

Figure 8. Fitted densities and probability plots for fatigue time of 101 6061-T6 aluminum coupons data

\section{Conclusion}

A new class of distributions called the WGPS family of distributions and its sub-class WGL distribution was developed. This new and large class of distributions and some of its structural properties are presented. Applications of the WLLoL, WWL and WBXIIL distributions to real datasets were given. We applied the model to real data sets to illustrate the applicability and usefulness of the proposed distribution. The special cases of the WLLoL, WWL and WBXIIL distributions were also compared to several non-nested models and found to provide the superior fit.

\section{Disclosure Statement}

The authors declare no conflict of interest.

\section{Acknowledgements}

The authors are very grateful to the editor and referees for reading an earlier version of this manuscript which led to this improved version.

\section{References}

Alamatsaz, M. H., \& Shams Harandi, S. (2016). Generalized Linear Failure Rate Power Series Distribution, Communications in Statistics - Theory and Methods, 45(8), 2204-2227. https://doi.org/10.1080/03610926.2013.878357

Barakat, H. M., \& Abdelkader, Y. H. (2004). Computing the Moments of Order Statistics from Nonidentical Random Variables, Statistical Methods and Applications, 13(1), 15-26. https://doi.org/10.1007/s10260-003-0068-9

Bourguignon, M., Silva, R. B., \& Cordeiro, G. M. (2014). A New Class of Fatigue Life Distributions, Journal of Statistical Computation and Simulation, 84(12), 2619-2635. https://doi.org/10.1080/00949655.2013.799164

Bourguignon, M., Silva, R. B., \& Cordeiro, G. M. (2014). The Weibull-G Family of Probability Distributions, Journal of Data Science, 12, 53-68.

Bidram, H., \& Nekoukhou, V. (2013). Double Bounded Kumaraswamy-Power Series Class of Distributions, Statistics 
and Operations Research Transactions, 37(2), 211-230.

Birnbaum, Z. W., \& Saunders, S. C. (1969). Estimation for a family of life distributions with applications to fatigue, Journal of Applied Probability, 6, 328-347.

Chambers, J., Cleveland, W., Kleiner, B., \& Tukey, P. (1983). Graphical Methods of Data Analysis, Chapman and Hall.

Chen, G., \& Balakrishnan, N. (1985). A General Purpose Approximate Goodness-of-fit Test, Journal of Quality Technology, 27(2), 154-161.

Cordeiro, G. M., \& Silva, R. B. (2014). The Complementary Extended Weibull Power Series Class of Distributions, Ciência e Natura, 36(3).

Cordeiro, G. M., Gomes, A., da Silva, C., \& Ortega, M. (2013). The Beta Exponentiated Weibull Distribution, Journal of Statistical Computation and Simulations, 38(1), 114-138.

Chahkandi, M., \& Ganjali, M. (2009). On some lifetime distributions with decreasing failure rate, Computational Statistics and Data Analysis, 53(12), 4433-4440.

Doostmoradi, A., Zadkarami, M. R., \& Roshani, S. A. (2014). A New Modified Weibull Distribution and Its Application, Journal of Statistical Research and Training Center, Iran 11, 97-118.

Flores, J., Borges, P., Cancho, V. G., \& Louzada, F. (2013). The Complementary Exponential Power Series Distribution, Brazilian Journal of Probability and Statistics, 27(4), 565584.

Gradshetyn, I. S., \& Ryzhik, I. M. (2000). Tables of Integrals, Series and Products, sixth edition, Academic Press, San Diego.

Hoskings, J. R. M. (1990). L-Moments: Analysis and Estimation of Distributions Using Linear Combinations of Order Statistics, Journal of the Royal Statistical Society, B 52, 105-124.

Johnson, N. L., Kotz, S., \& Balakrishnan, N. (1994). Continuous Distributions, Volume 1, John Wiley \& Sons, New York, NY.

Louzada, F., Marchi, V., \& Roman, M. (2014). The Exponentiated Exponential-Geometric Distribution: a Distribution with Decreasing, Increasing and Unimodal Failure Rate. Statistics, 48(1), 167-181.

Lu, W., \& Shi, D. (2011). A New Compounding Life Distribution: The Weibull-Poisson Distribution, Journal of Applied Statistics, 39(1), 21-38.

Morais, A. L., \& Barreto-Souza, W. (2011). A Compound Class of Weibull and Power Series Distributions, Computational Statistics and Data Analysis, 55(3), 1410-1425.

Mahmoudi, E., \& Jafari, A. A. (2012). Generalized Exponential-Power Series Distributions, Computational Statistics and Data Analysis, 56(12), 4047-4066.

Mahmoudi, E., \& Jafari, A. A. (2017). The Compound Class of Linear Failure Rate-Power Series Distributions: Model, Properties, and Applications, Communications in Statistics - Simulation and Computation, 46(2), 1414-1440.

Nadarajah, S., Popović, B. V., \& Ristić, M. M. (2013). Compounding: An R Package for Computing Continuous Distributions obtained by Compounding a Continuous and a Discrete Distribution, Computational Statistics, $28(3), 977-992$.

Nadarajah, S., \& Kotz, S. (2008). Strength Modeling using Weibull Distributions, Journal of Mechanical Science and Technology, 22(7), 1247-1254.

Oluyede, B. O., Huang, S., \& Pararai, M. (2014). A New Class of Generalized Dagum Distribution with Applications to Income and Lifetime Data, Journal of Statistical and Econometric Methods, 3(2), 125-151.

Oluyede, B. O., Warahena-Liyanage, G., \& Pararai, M. (2016). A New Compound Class of Log-logistic Weibull-Poisson Distribution: Model, Properties and Applications, Journal of Statistical Computation and Simulation, 86(7), 13631391.

Oluyede, B. O., Makubate, B., Fagbamigbe, A. F., \& Mdlongwa, P. (2018). A New Burr XII-Weibull-Logarithmic Distribution for Survival and Lifetime Data Analysis: Model, Theory and Applications, Stats 2018, 1(1), 77-91. https://doi.org/10.3390/stats1010006

Roman, M., Louzada, F., Cancho, V. G., \& Leite, J. G. (2012). A New Long-Term Survival Distribution for Cancer Data, Journal of Data Science, 10(2), 241-258.

Rodrigues, J. A., \& Silva, A. P. C. W. (2015). The Gamma-Dagum Distribution: Definition, Properties and Application, 
Mathematics and Statistics in Focus, 3(1), 1-7.

R Development Core Team (2014). A Language and Environment for Statistical Computing, R Foundation for Statistical Computing, Vienna, Austria

Silva, R. B., Bourguignon, M., Dias, C. R. B., \& Cordeiro, G. M. (2013). The compound class of extended Weibull power series distributions, Computational Statistics and Data Analysis, 58, 352.

Silva, R. B., \& Cordeiro, G. M. (2015). The Burr XII Power Series Distributions: A New Compounding Family, Brazilian Journal of Probability and Statistics, 29(3), 565-589.

\section{Copyrights}

Copyright for this article is retained by the author(s), with first publication rights granted to the journal.

This is an open-access article distributed under the terms and conditions of the Creative Commons Attribution license (http://creativecommons.org/licenses/by/4.0/). 